\title{
Non-Exclusive Financial Advice*
}

\author{
Salvatore Piccolo $^{\dagger} \quad$ Giovanni $^{\dagger}$. Puopolo P $^{\ddagger} \quad$ Luis Vasconcelos $^{\S}$
}

September 14, 2015

\begin{abstract}
We propose a simple model of non-exclusive financial advice in which two households rely on a self-interested (common) expert to make their investment choices. There is only one source of risk, and the expert is privately informed about the risky asset's volatility. When monetary transfers are unenforceable, we show that investors may delegate their investment decisions to the expert. When doing so, however, they impose restrictions on her choices which crucially depend on whether the expert perceives investors' asset allocations as complements or as substitutes. Finally, we analyze the implications of non-exclusivity in financial advice on investment behavior and welfare, and highlight a set of novel testable implications.
\end{abstract}

Keywords: Delegated Portfolio Management, Financial Advice, Non-Exclusivity

JEL Classification: G11 and G23

\section{Introduction}

In most developed countries money managers play an important role in households' investment decisions. A substantial portion of financial wealth is not managed directly by savers, but by specialized intermediaries. Moreover, investors often consult a financial advisor or a money manager before making any important financial decision. This propensity of investors to rely on financial advisors has been

\footnotetext{
*We thank Luca Colombo, Gianluca Feminis, Nicola Gennaioli, Emanuele Grassi, Nenad Kos, David Martimort, Debrah Meloso, Marco Ottaviani, Marco Pagano, Pierre Regibeau, Karl Schlag, and Emanuele Tarantino for helpful comments and discussions, as well as seminar participants at University of Bergen (NHH), the Catholic University of Milan, the CERGE-EI (Prague), the University of Essex, the University of Wien, and conference attendees at the 2014 European meeting of the Econometric Society, the 2014 Conference of the European Association for Research in Industrial Economics, and the Industrial Organization workshop in Alberobello in 2014.

${ }^{\dagger}$ Dipartimento di Economia e Finanza, Universita Cattolica del Sacro Cuore, Via Necchi 5, 20123 Milan, Italy. E-mail: salvapiccolo@gmail.com

${ }^{\ddagger}$ Department of Finance, Bocconi University, Via Roentgen 1, Milan, Italy, CSEF, BaffiCarefin Center. E-mail: giovanni.puopolo@unibocconi.it

${ }_{\S}^{\S}$ Department of Economics, University of Essex, Wivenhoe Park, Colchester, Essex CO4 3SQ, United Kingdom. E-mail: lvasco@essex.ac.uk
} 
documented in recent empirical studies. A survey conducted by Hung et al. (2008) reveals that 73 percent of all US retail investors consult a financial advisor before purchasing shares. In a large online survey among recent purchasers of investment products in the EU, Chater et al. (2010) found that nearly 80 percent made their purchases through an intermediary, with 58 percent of them claiming that an advisor influenced their choice.

Many existing theories explain why, and under which conditions, investors delegate to experts their portfolio choices. However, most of these models focus on the relationship between an investor and his exclusive advisor, whereas little is known on how investors should behave when they interact with an expert who simultaneously advises other investors. What type of externalities do investors exert on each other when they share the same advisor? Do experts tend to induce excessive risk-taking when they advise multiple clients? How does non-exclusivity in financial advice affect investors' asset-allocation behavior and welfare?

We address these issues by examining the mechanism design problem that an investor faces when he is advised by an expert who also deals with other investors. Non-exclusivity in the financial advice matters to each individual investor because the expert's behavior with an investor may depend on her relationships with other investors. For example, if some clients decide to invest heavily in a particular product, the advisor's incentive to induce her other clients to invest in that product may soften or strengthen depending on whether some sale targets have been reached or not.

We analyze a simple model of non-exclusive financial advice in which an expert advises two identical investors who desire to invest their money into a risky asset, but do not have enough knowledge to do it personally. The expert has private information about the riskiness of this asset - i.e., the state of the world. Investors cannot provide incentives to the advisor by means of money transfers. Both this assumption and the fact that financial advice is non-exclusive seem particularly compelling for the case of small investors (e.g., households). Indeed, when making their investment decisions, households are usually advised by their banks' employees, whose services are typically non-exclusive and do not require additional costs over and above the (fixed) fees required to open a deposit account. Accordingly, each investor chooses and commits to an asset allocation rule (mechanism) that maps the advisor's report about the state of the world into a portfolio choice. We characterize the investment rules chosen by investors in equilibrium of this common agency game and determine the conditions under which these outcomes feature 'delegated portfolio management' and when, instead, clients prefer to enforce rigid investment rules that are unresponsive to the information reported by the advisor. The objective is to understand how non-exclusivity in financial advice alters experts' incentive to induce excessive risk-taking, the form of delegation that arises in equilibrium, and its implications for welfare.

To gain insights on the novel forces that non-exclusivity in financial advice brings out, we first consider the benchmark in which the advisor deals with only one client - i.e., the case of exclusive 
financial advice. Following the earlier literature, we focus on the case in which the advisor wants her client to overinvest in the risky asset relative to the first-best rule. ${ }^{1}$ In this case, the advisor has an incentive to claim that the riskiness of this asset is lower than its actual value. The investor takes into account this partisan bias when designing the optimal investment rule. The solution of his mechanism design problem consists in delegating the investment decision to the advisor, but imposing a cap on the amount the advisor can allocate to the risky asset - i.e., a partial delegation strategy. On the one hand, by delegating to the expert his asset-allocation choice, the investor incorporates the relevant private information of the advisor in the composition of his portfolio. On the other hand, the imposition of a cap enables him to cope with the advisor's bias towards an overly risky choice. The optimal cap balances the benefit from using the advisor's private information and the cost stemming from the latter's partisan bias.

Next, we examine the case of non-exclusive financial advice. With a common expert, the portfolio choice of one client may affect the expert's ideal investment choice of the other client. We find that whether the advisor perceives her clients' portfolio choices as substitutes or as complements plays a key role in the determination of the mechanisms chosen by the investors in equilibrium. Investment choices are perceived as substitutes when the advisor's incentive to induce a higher risk exposure by one client weakens as the other client's risk exposure increases. In contrast, investment choices are perceived as complements when the advisor's incentive to induce a higher risk exposure by one client strengthens when the risk exposure of the other client increases too. In reality, whether portfolio choices are perceived as substitutes or as complements by a financial advisor depends on her indirect utility function, which originates both from her preference structure (e.g., risk aversion, reputational and career concerns and so on) and the incentive scheme to which she is exposed by her employer (for example a bank). In the baseline model, for the sake of tractability, we take this indirect utility function as given. However, in the extensions, we show that substitutability may be driven by the expert's reputational and career concerns, which emerge for example when the advisor is fired by the clients if the investment does not perform sufficiently well, or by the advisor's need to hedge income risk when she is risk averse. Instead, complementarity may result from sales incentives, granted to the bank by the provider of the financial product, which are convex in the total amount of sales. Since none of these cases seems to be more realistic than the other, both are considered in the analysis.

When the financial advisor perceives investors' portfolio choices as complements she has an incentive to induce each investor to take even more risk than in the case of exclusive financial advice. As a result, investors trust less the expert and delegate less - i.e., their investment choices are less responsive to the type of asset they buy and, as long as some delegation occurs, it happens only when investors buy very risky assets. Overall, the fact that portfolio allocations are perceived as complements hinders the

\footnotetext{
${ }^{1}$ See, e.g., Inderst and Ottaviani (2012b), Stracca (2005) and Ottaviani (2000).
} 
relationship between the advisor and each investor relative to the exclusivity case. In other words, investors generate a negative externality on each other: their expected utility is higher under exclusive financial advice.

In contrast, when the expert perceives investors' portfolio choices as substitutes she has an incentive to induce each client to take less risk than in the case of exclusive financial advice. This mitigates the conflict of interests between the investors and the financial advisor, but only up to a certain point. When the degree of substitutability is low, investors trust more the financial advisor and delegate more by increasing the cap on the proportion of their wealth the advisor can allocate to the risky asset. In this case, the presence of one investor generates a positive externality on the other investor. Investors are better off when financial advice is non-exclusive than when it is exclusive. However, when the degree of substitutability is sufficiently high, the direction of the misalignment of incentives changes. In this case, the advisor has an incentive to induce investors to allocate too little wealth into the risky asset relative to their first-best allocation ${ }^{2}$, thereby generating a conflict of interest of a different sort. In equilibrium, investors respond by changing the investment rule they propose to the financial advisor. Specifically, they still delegate the portfolio decision to the financial advisor, but now impose a floor on the proportion of their wealth that is invested in the risky asset. Hence, with strong substitutability delegation occurs only when investors buy low risk products. Clearly, whether investors are better off than in the case of exclusive financial advice depends on the degree of substitutability. The higher the substitutability, the larger the misalignment of incentives, the less investor delegate by imposing a higher floor and the lower the investors' expected utility.

These results are qualitatively robust to a number of extensions that include multiple dimensions of asymmetric information between the advisor and the investors, more than two investors, and heterogeneity of investors. Taken together, they offer novel insights on the rules according to which investors would like to regulate their relationships with financial experts when advice is non-exclusive. Our analysis suggests that experts may not always induce excessive risk taking, and that the extent to which this occurs hinges on the interplay between the structure of the compensation schemes to which they are exposed, their reputational and career concerns. Moreover, by affecting the optimal design of delegation rules for money managers, this relationship impacts investors' expected utility in a non-obvious manner, and therefore it shapes the social desirability of regulatory rules that determine sales commissions, how experts weight their reputation and hedge income risk.

Interestingly, even if there is no direct evidence on the 'correlation' between non-exclusivity, investor's propensity to delegate, the characteristics of the products they buy and their risk exposure, the emergence of equilibria with partial delegation, whose features do not depend exclusively on investors' characteristics, seems consistent with the evidence collected by Foerster et al. (2014) who find that

\footnotetext{
${ }^{2}$ That is, the allocation that would arise in case the investor is fully informed about the state of the world.
} 
clients' observable characteristics jointly explain only $11 \%$ of the variation in risky share in the crosssection of Canadian households. They argue that advisor fixed effects have substantial explanatory power and impute this evidence to the advisors' idiosyncratic 'tastes' in portfolio allocation, which may reflect agency conflicts similar to those studied throughout our paper. This evidence should be interpreted with care though. Indeed, Foerster et al. (2014) do not account explicitly for the non-exclusive dimension of financial advice, although by considering retail investors they are quite likely restricting attention to deals that are non-exclusive.

The paper is structured as follows. In Section 2 we review the relevant literature and highlight our contribution. Section 3 lays down the baseline model with symmetric investors. Section 4 studies the exclusivity benchmark. In Section 5 we characterize equilibria with non-exclusive financial advice. In Section 6 we provide the comparative statics analysis and compare the regimes with and without exclusive advice. In Section 7, we show how the advisor's career concerns, risk aversion and the shape of sales' commissions may lead her to perceive her clients' portfolio choices as substitutes or as complements and consider several extensions of the basic model. Section 8 reviews the model's main empirical predictions and concludes. All proofs are in the Appendix.

\section{Related literature}

The agency problem between investors and their money managers has been extensively studied by the earlier literature - see, e.g., Stracca (2005) and Inderst and Ottaviani (2012b) for recent surveys of this literature. Existing models highlight many important aspects of standard delegated portfolio management by studying how investors should optimally design contracts (remuneration schemes) for money managers. Following the moral-hazard tradition some of these models assume that the money manager chooses the riskiness and/or the expected return of his client's portfolio, and that this choice is unobservable to the investor, who then needs to design a second-best contract that motivates the expert to choose the right action (e.g., Adamati and Pfleiderer, 1997; Stoughton, 1993; Palomino and Prat, 2003; and Palomino and Uhlig, 2006). In the adverse selection framework, Allen (1985) and Bhattacharya and Pfleiderer (1985) are the first to propose models where a better informed advisor must be solicited to reveal superior information about the rate of return and/or the riskiness of a financial asset to an uninformed investor. These papers show that optimal contracts do not achieve the first-best solution due to the standard trade-off between efficiency and information rents (see also Allen and Gorton, 1993; and Das and Sundaram, 1998). Similar points are made in the literature studying the link between complexity and obfuscation. For example, Carlin and Manso (2010) show that when investors are uninformed, they may either pay excessive fees or invest in 'bad assets'. Hence, providers of financial products earn higher rents from investors who are unsophisticated and more uninformed. 
Our paper follows the adverse selection approach. But, in contrast with earlier models, we posit that money transfers between investors and their advisors are not feasible. We share this approach both with the recent bulk of work on optimal delegation pioneered by Holmstrom (1984) — see, e.g., Alonso and Matusheck (2008), Dessein (2002), Martimort and Semenov (2006), Melumad and Shibano (1996), for abstract delegation settings, and Morgan and Stocken (2003), among others, for an application to stock recommendation. Our novel contribution to both these literatures is the non-exclusivity aspect. ${ }^{3}$ So far, no delegation models have accounted for this possibility with the exception of Asparouhova et al. (2013) who also model delegated portfolio management as non-exclusive, but take a general equilibrium approach. In their model managers compete to attract investors by offering bundles of portfolio allocations and intermediation fees, investors can buy at linear prices any combination of portfolios they want. By looking at the general equilibrium implications of non-exclusive advice, they offer a number of interesting predictions (that are then tested through an experiment) on the way competitive money managers should behave both on the pricing and product design sides. However, in their model there is no asymmetric information: delegated portfolio management is not an endogenous result, but rather an assumption. In this sense, our models are complementary.

Finally, notice that in our model the investors' propensity to rely on the financial advisor is an equilibrium phenomenon - i.e., investors optimally decide to delegate their portfolio choices to the advisor balancing out the costs and benefits of leaving discretion to the expert. This is different from the approach taken in Gennaioli et al. (2013) where trust is modeled as a behavioral attitude that reduces the investors' perception of the riskiness of a given investment. In this sense, our models complement one another. Specifically, while Gennaioli et al. (2013) focuses on the implications of trust on asset allocation choices, our mechanism design approach abstracts from the issue of modeling preferences that are trust dependent, and focuses on the implications of asymmetric information on the investors' propensity to rely on financial advice.

\section{The baseline model}

Players and environment. Consider two identical investors (each denoted by $i=1,2$ ) with initial wealth normalized to 1 . There is only one risky investment opportunity (e.g., equities, funds, structured products and so on) and the riskless asset (e.g., government bonds, pension and insurance plans, etc.). The stochastic return of the risky asset $\tilde{r}$ has mean $\mu$ and variance $\sigma^{2}$. The riskless asset pays the riskfree rate $r_{f}$, with $\mu>r_{f} \geq 1$.

Due to the lack of proper financial education, investors must rely on a (common) financial advisor to make their investment choices. The advisor is better informed than the investors about the variance $\sigma^{2}$

\footnotetext{
${ }^{3} \mathrm{~A}$ similar approach is taken in the cheap talk literature by Farrel and Gibbons (1989) who propose a model in which a sender deals with two receivers but there are no externalities between the receivers' actions.
} 
of the risky investment (the state of the world). More precisely, while the expected return $\mu$ is common knowledge, the variance $\sigma^{2}$ cannot be assessed with certainty by the investors who need to rely on the superior knowledge of the expert. In the absence of financial advice, investors have only a symmetric prior about the state of the world: they correctly believe that $\sigma^{2}$ distributes uniformly over the compact support $\Sigma \equiv[1-\Delta, 1+\Delta]$, with $\Delta \in(0,1) .{ }^{4}$ This prior is common knowledge.

Preferences and conflict of interests. Investors are risk averse with respect to wealth. Their interim expected utility exhibits mean-variance preferences - i.e., for a given state of the world $\sigma^{2}$, the utility of an investor $i$ who allocates $\alpha_{i}$ of his wealth to the risky asset is

$$
u\left(\alpha_{i}, \sigma^{2}\right)=\mathbb{E}\left[\alpha_{i} \tilde{r}+\left(1-\alpha_{i}\right) r_{f}\right]-\frac{\gamma}{2} \mathbb{E}\left[\alpha_{i}(\tilde{r}-\mu)\right]^{2}=\alpha_{i}\left(\mu-r_{f}\right)+r_{f}-\frac{\gamma}{2} \alpha_{i}^{2} \sigma^{2},
$$

where $\gamma>0$ measures the investor's coefficient of risk aversion. Hence, absent asymmetric information, the investors' optimal asset allocation yields the first-best benchmark — i.e., the standard mean-variance allocation

$$
\alpha^{F}\left(\sigma^{2}\right)=\frac{\mu-r_{f}}{\gamma \sigma^{2}}
$$

At $\sigma^{2}=1$, the first-best asset allocation $\alpha^{F}(1)$ can be interpreted as a measure of the risk premium per unit of risk aversion. To simplify exposition, we will define this index by $\pi \equiv \alpha^{F}(1)$.

By contrast, when investors do not know the state of the world $\sigma^{2}$ and do not rely on the expert, their unconditional expected utility is

$$
\int_{\sigma^{2}} u\left(\alpha_{i}, \sigma^{2}\right) \frac{d \sigma^{2}}{2 \Delta}=\alpha_{i}\left(\mu-r_{f}\right)+r_{f}-\frac{\gamma}{2} \alpha_{i}^{2} \mathbb{E}\left[\sigma^{2}\right]
$$

which is maximized at $\alpha_{i}^{*}=\pi$.

The advisor's preferences are represented by the following quadratic loss function that depends

\footnotetext{
${ }^{4}$ The idea is that non-institutional investors, such as households, with limited access to detailed information on asset returns, are less able to quantify the risk carried by financial activities than their expected returns. Palomino and Uhlig (2007), for instance, argue that mutual fund regulation does not require funds to disclose their portfolio very often, and managers window dress around disclosure dates. Therefore, for young funds with a short track record, estimating the return volatility may not be possible in the absence of insider information. Moreover, if one considers private equity funds, their return volatility may be difficult to estimate since their net asset value is not very often observed. The idea is that non-institutional investors, such as households, with limited access to detailed information on asset returns, are less able to quantify the risk carried by financial activities than their expected returns. Palomino and Uhlig (2007), for instance, argue that mutual fund regulation does not require funds to disclose their portfolio very often, and managers window dress around disclosure dates. Therefore, for young funds with a short track record, estimating the return volatility may not be possible in the absence of insider information. Moreover, if one considers private equity funds, their return volatility may be difficult to estimate since their net asset value is not very often observed. Alternatively, one can imagine that unsophisticated investors find it harder to estimate the return volatility of an asset than to estimate its expected return. This may be because the former estimate relies on the latter.
} 
symmetrically on the investors' portfolio choices - i.e.,

$$
v\left(\boldsymbol{\alpha}, \sigma^{2}\right)=-\frac{1}{2} \sum_{i=1,2}\left[\alpha_{i}-(1+\lambda) \alpha^{F}\left(\sigma^{2}\right)\right]^{2}-\theta \alpha_{1} \alpha_{2}
$$

where $\boldsymbol{\alpha} \equiv\left(\alpha_{1}, \alpha_{2}\right){ }^{5}$ The parameter $\lambda \in[0,1)$ in the first term of this equation reflects the strength of the conflict of interests between the expert and each client. As argued in Ottaviani (2000), this misalignment of preferences may be the result of (linear) commissions paid to the financial advisor for selling some types of financial products. ${ }^{6}$

The second term, instead, captures the potential effect of the decision of one investor on the common advisor's behavior when she interacts with other investors. In other words, it reflects the potential externalities that investors may cause on one another through the advisor's preferences. The parameter $\theta \in(-1,1)$ measures the direction and magnitude of such externalities. Observe that $\partial^{2} v(\cdot) / \partial \alpha_{1} \partial \alpha_{2}=$ $-\theta$. Hence, $\theta<0$ captures the case where the investors' portfolio choices are perceived as complements by the advisor: she prefers investor $i$ to allocate a larger fraction of his wealth into the risky activity when investor $j$ does so too. This complementarity may arise for example because providers of the financial products pay commissions to the bank that reward not only the achievement of investment targets on each single client, but that also award premia that are increasing with the size of the clientele gathered by the bank - e.g., when funds reward the achievement of clients' targets.. In contrast, $\theta>0$ captures the case where the investors' portfolio choices are perceived as substitutes by the advisor: she wants investor $i$ to allocate a lower fraction of his wealth in the risky asset when investor $j$ 's investment in that asset increases. This may for instance reflect her concerns about reputational losses following unsuitable sales. ${ }^{7}$

In Section 7.1 we show more carefully how these externalities might result from the advisor's sales commissions, and her reputational and career concerns. Of course, when $\theta=0$, the advisor's utility is separable across clients, and there is no difference between exclusive and non-exclusive financial advice.

Contracting. Contracts that require state contingent monetary transfers between the advisor and her clients are not enforceable - i.e., there cannot be fees contingent on the information transmitted by the expert to the investors (fixed fees are normalized to zero without loss of generality). Most commonly, in fact, clients pay no direct compensation to advisors for their services. Hence, we focus on a simple class of (bilateral) direct mechanisms such that each investor $i$ chooses a direct mechanism $\mathcal{M}_{i} \equiv\left\{\alpha_{i}\left(m_{i}\right)\right\}_{m_{i} \in \Sigma}$, with $\alpha_{i}(\cdot): \Sigma \rightarrow \Re$, which specifies a portfolio allocation $\alpha_{i}\left(m_{i}\right)$ for any (private)

\footnotetext{
${ }^{5}$ Similar loss functions are used in the cheap-talk literature (e.g., Crawford and Sobel, 1982).

${ }^{6}$ An analogous approach is followed in Stocken (2003).

${ }^{7}$ Gennaioli et al. (2013), for instance, argue that money mangers are particularly sensitive to their reputation. In our model we are implicitly assuming that reputation may be crucially affected by the size of an advisor's clientele via network effects that may arise from (un-modeled) information spillovers between clients.
} 
report $m_{i} \in \Sigma$ made by the advisor to investor $i$ about the state of the world $\sigma^{2}$. As standard in this literature, mechanisms are restricted to be continuous and piecewise differentiable. ${ }^{8}$ The expert cannot refuse advice to his clients: an intrinsic common agency game - i.e., a game in which the common agent cannot refuse to deal with some of her principals. ${ }^{9}$

The use of direct mechanisms allows us to rely on intuitive and easy to characterize incentive constraints. Yet, as we will see in Section 5.3, all the equilibrium outcomes obtained throughout the paper can be implemented by simple indirect mechanisms that require only each investor to announce a choice set from which the advisor can pick his preferred allocation.

Timing. The timing of the game is as follows:

- Period 0: Nature draws $\sigma^{2}$ and only the advisor observes its realization.

- Period 1: Each investor $i$ announces a mechanism $\mathcal{M}_{i}$ to the advisor and commits to it. These announcements are simultaneous.

- Period 2: The advisor (privately) reports $m_{i}$ to each investors $i$. Investment choices are made according to the mechanisms chosen in Period 1.

- Period 3: Asset returns materialize.

The assumption that investors can commit to the decision rule they announce is standard in the mechanism design literature that studies delegation in the absence of monetary incentives. Moreover, it allows us to disentangle the effects of asymmetric information on equilibrium portfolio choices from those due to lack of commitment, and to avoid the typical selection issue of cheap talk games (e.g., Crawford and Sobel, 1982, among others). This hypothesis is often motivated with a reputation argument: the relationship between an investor and his financial advisor is usually long-lasting (due to switching costs). In addition, in Section 5.3 we argue that our equilibrium mechanisms are robust to the threat of renegotiation as long as appropriate disinvestment fees can be enforced.

We make the following assumption on the parameters of the model.

\footnotetext{
${ }^{8}$ It should be noted that, more generally, these mechanisms may exhibit discontinuities similar to those that arise with the partition equilibria of signaling models a' la Crawford and Sobel (1982). However, as shown in Martimort and Semenov (2006) in a single principal-agent relationship, continuous mechanisms are optimal whenever the distribution function of the agent's type is strictly log-concave. Yet, although the uniform distribution satisfies such requirement, in our model the analysis may be slightly more complex due to the common agency dimension: best replies to discontinuous mechanisms may not be continuous mechanisms. Yet, using the approach developed by Martimort and Semenov (2006) one can show that a best reply to a continuous mechanism must be a continuous mechanism as long as the strict log-concavity requirement is met. Addressing the issue of the existence of equilibria with discontinuous mechanisms is out of the scope of this paper.

${ }^{9}$ Investors could offer more complex mechanisms where their portfolio allocation depends not only on the advisor's report about the state of the world, but also on the mechanism offered to the advisor by the other investor. While it is realistic to consider this type of mechanisms in some contexts (see, e.g., Attar et al., 2011; Martimort and Stole, 2002 and 2003; and Pavan and Calzolari, 2009), we believe it is less so in our environment and do not consider them.
} 
Assumption 1. $1-\lambda+2 \theta>0$ and $\pi<1-\Delta$.

This assumption simplifies the analysis as it implies that taking short positions on any of the two assets is never optimal - i.e., under the optimal mechanism $0 \leq \alpha_{i} \leq 1$ for $i=1,2$. Finally, we assume that the structure of the advisor's preferences is common knowledge - i.e., both investors know $\lambda$ and $\theta$. This means that investors are wary of the conflict of interests with the advisor. In Section 7.4 we discuss the case where the precise magnitude of these conflict of interests is not known to investors.

We use Perfect Bayesian Equilibrium as the equilibrium concept.

\section{The exclusivity benchmark}

We begin with the analysis of the case in which the advisor provides financial advice to one investor only. The analysis of this benchmark provides basic insights on the trade-offs that determine the equilibrium portfolio choices in our context.

Since the advisor's risk preferences are misaligned with those of the investor - i.e., $\lambda>0-$ the expert has an incentive to manipulate her report about the state of the world. To prevent this behavior, the optimal mechanism must elicit truthful information revelation. Given a mechanism $\alpha(\cdot)$, the advisor's utility is

$$
v\left(\alpha(m), \sigma^{2}\right) \equiv-\frac{1}{2}\left[\alpha(m)-(1+\lambda) \alpha^{F}\left(\sigma^{2}\right)\right]^{2},
$$

when she reports $m$ to the investor and the state of the world is $\sigma^{2}$. Within the class of continuous mechanisms, incentive compatibility requires ${ }^{10}$

$$
\frac{\partial}{\partial m} v\left(\alpha\left(\sigma^{2}\right), \sigma^{2}\right)=0 \quad \Leftrightarrow \quad\left[\alpha\left(\sigma^{2}\right)-(1+\lambda) \alpha^{F}\left(\sigma^{2}\right)\right] \dot{\alpha}\left(\sigma^{2}\right)=0
$$

Essentially, the asset allocation rule announced by the investor must be such that the advisor's utility is maximized when she truthfully reports the state of the world. ${ }^{11}$ Condition (4.1) is satisfied by two interesting classes of asset allocation rules: the pooling ones, where the asset allocation is unresponsive to the state of the world - i.e., when the derivative $\dot{\alpha}\left(\sigma^{2}\right)$ is equal to zero; and the separating ones that mandate an asset allocation that coincides with the advisor's ideal point - i.e.,

$$
\alpha_{E}^{A}\left(\sigma^{2}\right)=(1+\lambda) \alpha^{F}\left(\sigma^{2}\right)
$$

This last type of rule can be seen as delegation by the client of the investment decision to the advisor. An optimal investment rule for the investor might also combine the two schemes, so that for some

\footnotetext{
${ }^{10}$ See, e.g., Alonso and Matusheck (2008), Martimort and Semenov (2006), and Melumad and Shibano (1991).

${ }^{11}$ Direct inspection of the incentive compatibility constraint (4.1) reveals why in our framework fixed fees are irrelevant and can be normalized to zero without loss of generality - they have no effect on the advisor's incentive to misreport the true state of the world.
} 
subsets of $\Sigma$ it is optimal to impose a fixed allocation and for others it is optimal to let the advisor pick her most preferred asset allocation.

Let $\mathcal{P}=\cup_{k=1}^{K} \mathcal{P}_{k}$ be the union of all $K$ subsets of $\Sigma$ in which the investor pools by choosing $\alpha_{k}$ for every $\sigma^{2} \in \mathcal{P}_{k}$. The investor's maximization problem can be written as

$$
\max _{\left(\mathcal{P}_{k}, \alpha_{k}\right)_{k=1}^{K}, K}\left\{\int_{\Sigma \backslash \mathcal{P}} \alpha_{E}^{A}\left(\sigma^{2}\right)\left[\pi-\frac{\alpha_{E}^{A}\left(\sigma^{2}\right) \sigma^{2}}{2}\right] d \sigma^{2}+\sum_{k=1}^{K} \int_{\mathcal{P}_{k}} \alpha_{k}\left[\pi-\frac{\alpha_{k} \sigma^{2}}{2}\right] d \sigma^{2}\right\}
$$

subject to $K \in \mathbb{Z}, \mathcal{P}_{k} \subseteq \Sigma$ and $\alpha_{k} \in[0,1]$ for every $k=1, . ., K{ }^{12}$

Proposition 1. The optimal mechanism for the investor when financial advice is exclusive, $\mathcal{M}_{E}^{*} \equiv$ $\left\{\alpha_{E}^{*}(m)\right\}_{m \in \Sigma}$, satisfies the following properties:

- If $\lambda<\Delta$, then the investor partially delegates the investment decision to the financial advisor. Specifically, his investment decision is given by

$$
\alpha_{E}^{*}\left(\sigma^{2}\right)= \begin{cases}\alpha_{E}^{*} & \text { if } \quad \sigma^{2} \leq x_{E}^{*} \\ \alpha_{E}^{A}\left(\sigma^{2}\right) & \text { if } \quad \sigma^{2}>x_{E}^{*}\end{cases}
$$

with $x_{E}^{*}=\frac{1+\lambda}{1-\lambda}(1-\Delta) \in(1-\Delta, 1+\Delta)$ and

$$
\alpha_{E}^{*}=\frac{\pi}{\mathbb{E}\left[\sigma^{2} \mid \sigma^{2} \leq x_{E}^{*}\right]}=\pi \frac{1-\lambda}{1-\Delta} .
$$

- If $\lambda \geq \Delta$, then the investor totally ignores the information provided by the financial advisor and invests $\alpha_{E}^{*}\left(\sigma^{2}\right)=\pi$ for all $\sigma^{2} \in \Sigma$.

The optimal mechanism is shaped by two contrasting forces. To induce a truthful report by the advisor, the investor must either force a pooling allocation, or he must allow the advisor to obtain her ideal investment choice. Both schemes depart from the investor's first best allocation, and are thus costly to him. On the one hand, an investment rule unresponsive to the state of the world - i.e., the pooling one - is costly to the investor because he is risk averse, and thus would like to invest an amount of wealth into the risky asset tailored to its return volatility. On the other hand, the cost of linking the investment strategy to the state of the world - i.e., a separating outcome - stems for the fact that such allocation must coincide with the advisor's ideal investment to guarantee truthful information revelation. But, because preferences are misaligned, this carries more risk than what the investor would like to bear.

The relative magnitude of these costs determines the structure of the optimal asset allocation. Proposition 1 states that, when the conflict of interest between the investor and his advisor is not

\footnotetext{
${ }^{12}$ Hereafter the symbol $\mathbb{Z}$ will denote the set of all integers.
} 
very strong $(\lambda<\Delta)$, it is optimal for the investor to leave discretion to the advisor and enable her to implement her ideal point if the volatility of the risky asset is larger than the threshold $x_{E}^{*}$. This is because the difference between the players' ideal points is less pronounced when the realized variance $\sigma^{2}$ is large. ${ }^{13}$ Hence, the cost of delegation is relatively less severe than the cost of pooling to the investor, who prefers a portfolio that covaries with the state of the world. By contrast, when $\sigma^{2}$ is low, the agency conflict is harder to be reconciled with a separating allocation: in these states of the world the advisor's most preferred investment into the risky asset is much larger than that of the investor. Thus, it is optimal for the latter to force a flat rule, which as condition (4.2) shows, coincides with the first-best rule when the investor only knows that $\sigma^{2}$ distributes between $1-\Delta$ and $x_{E}^{*}$. Notice, however, that when $\lambda$ is large enough, the objectives of the two players diverge so much that the cost of delegation always outperforms that of basing financial decisions on the prior alone. In this case, the optimal asset allocation rule requires a fully pooling allocation - i.e., $x_{E}^{*}=1+\Delta$.

Figure 1 below, provides a graphical representation of the optimal asset allocation with partial delegation:

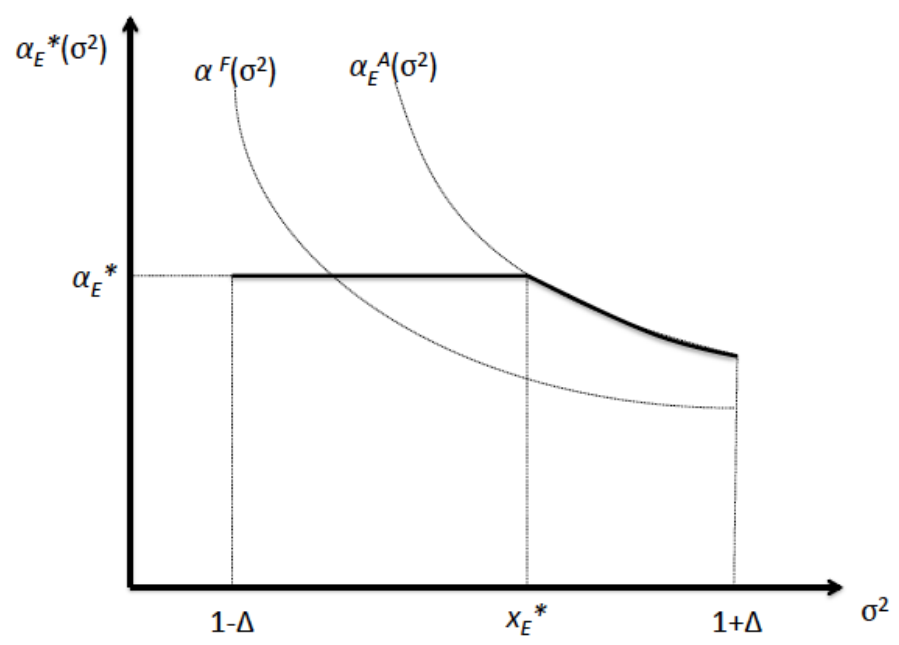

Figure 1: Delegation under exclusive financial advice.

The optimal asset allocation rule is represented by the solid line, which corresponds to the advisor's ideal point for all $\sigma^{2}$ larger than $x_{E}^{*}$, and features a cap for all $\sigma^{2}$ lower than $x_{E}^{*}$.

Hence, under exclusivity, uninformed investors rely more often on financial advise when buying assets whose return volatility is large. Another interesting prediction is that, compared to the first-best benchmark, there is under-investment into the risky activity when its return volatility is low, and overinvestment otherwise. In addition, as expected, the pooling region expands (i.e., $x_{E}^{*}$ increases) when $\lambda$ becomes larger because the misalignment of preferences between the investor and the advisor becomes more severe. The impact of the risk premium and the risk aversion coefficient on the optimal portfolio

\footnotetext{
${ }^{13}$ That is, the difference $\alpha_{E}^{A}\left(\sigma^{2}\right)-\alpha^{F}\left(\sigma^{2}\right)=\lambda \pi / \sigma^{2}$ is decreasing in $\sigma^{2}$.
} 
allocation are as in the standard mean-variance analysis. What is perhaps less obvious is the impact of $\lambda$ on the optimal portfolio allocation. Clearly, a larger intrinsic bias $\lambda$ increases the optimal (risky) investment within the delegation region. But, the opposite holds true in the pooling region. In this case, a larger $\lambda$ reduces the pooling allocation because it exacerbates the conflict of interest between the advisor and the investor. This leads the latter to adopt a more conservative investment strategy in order to reduce his exposure to the former's opportunistic behavior. Finally, it can be easily seen that a larger $\Delta$ induces delegation in a larger set of circumstances ${ }^{14}$ : less transparency or poor financial literacy of investors induce more reliance on money managers. This also explains why the investment into the risky asset in the pooling region becomes larger when uncertainty increases - i.e., $\alpha_{E}^{*}$ increases with $\Delta$.

\section{Non-exclusive financial advice}

Suppose now the advisor deals simultaneously with two identical investors. We begin by analyzing how non-exclusivity of the financial advice modifies the advisor's incentive compatibility constraint and her ideal investment choices.

Given mechanisms $\alpha_{1}(\cdot)$ and $\alpha_{2}(\cdot)$, the advisor' utility if he reports $m_{1}$ and $m_{2}$ to investors when the true variance is $\sigma^{2}$ is given by

$$
v\left(\alpha_{1}\left(m_{1}\right), \alpha_{2}\left(m_{2}\right), \sigma^{2}\right)=-\frac{1}{2} \sum_{i=1,2}\left[\alpha_{i}\left(m_{i}\right)-(1+\lambda) \alpha^{F}\left(\sigma^{2}\right)\right]^{2}-\theta \alpha_{1}\left(m_{1}\right) \alpha_{2}\left(m_{2}\right) .
$$

Using the same logic as before, the (local) incentive compatibility conditions, necessary and sufficient to guarantee that the advisor truthfully reports $\sigma^{2}$ to each investor are

$$
\frac{\partial}{\partial m_{i}} v\left(\alpha_{1}\left(\sigma^{2}\right), \alpha_{2}\left(\sigma^{2}\right), \sigma^{2}\right)=0 \quad \Leftrightarrow \quad\left[\alpha_{i}\left(\sigma^{2}\right)-(1+\lambda) \alpha^{F}\left(\sigma^{2}\right)+\theta \alpha_{j}\left(\sigma^{2}\right)\right] \dot{\alpha}_{i}\left(\sigma^{2}\right)=0,
$$

for $i=1,2$. Hence, for any given mechanism $\alpha_{j}\left(\sigma^{2}\right)$ chosen by investor $j$, an incentive compatible mechanism for investor $i$ is either flat - i.e., $\dot{\alpha}_{i}\left(\sigma^{2}\right)=0$ - or it requires

$$
\alpha_{i}\left(\sigma^{2}\right)=\alpha_{E}^{A}\left(\sigma^{2}\right)-\theta \alpha_{j}\left(\sigma^{2}\right) \equiv \alpha_{i}^{A}\left(\sigma^{2}\right)
$$

which is the advisor's ideal point on investor $i$ 's portfolio choice (given the choice of investor $j$ ).

Equation (5.1) highlights the key feature of the case where the expert advises more than one investor: her ideal point on investor $i$ is equal to the individual (or intrinsic) target $\alpha_{E}^{A}\left(\sigma^{2}\right)$ net of the externality

\footnotetext{
${ }^{14}$ Indeed, the area where the investor forces a pooling allocation, which is measured by the ratio $\frac{x_{E}^{*}-(1-\Delta)}{2 \Delta}=$ $\frac{1-\Delta}{\Delta} \frac{\lambda}{1-\lambda}$, shrinks when $\Delta$ increases.
} 
$\theta \alpha_{j}\left(\sigma^{2}\right)$. Hence, other things being equal, the expert's incentive to understate or overstate the value of $\sigma^{2}$ depends both on the sign and the magnitude of the interaction parameter $\theta$.

Noteworthy, if $\dot{\alpha}_{i}\left(\sigma^{2}\right) \neq 0$ for each $i=1,2$, then it must be the case that both investors choose the same asset allocation

$$
\alpha_{N}^{A}\left(\sigma^{2}\right)=\frac{\alpha_{E}^{A}\left(\sigma^{2}\right)}{1+\theta}
$$

which is (strictly) decreasing in $\sigma^{2}$. Essentially, if both investors fully delegate their portfolio choices to the advisor, they invest the same fraction of wealth into the risky asset. This is because the advisor's preferences are symmetric with respect to investment choices, and investors feature the same attitude towards risk.

Since investors are identical we focus on symmetric equilibria in which they choose the same mechanism $\mathcal{M}_{N}^{*}$, which requires pooling in the subset $\mathcal{P}_{N}^{*} \subseteq \Sigma$ and, within this subset, allocates a share $\alpha_{N}^{*}$ of wealth to the risky asset. The mechanism $\mathcal{M}_{N}^{*}$ must solve the maximization problem of each investor given that the other investor also chooses $\mathcal{M}_{N}^{*}$. Let $\mathcal{P}_{i}=\cup_{k=1}^{K_{i}} \mathcal{P}_{i, k}$ be the union of all $K_{i}$ subsets of $\Sigma$ in which investor $i$ pools by choosing $\alpha_{i, k}$ for every $\sigma^{2} \in \mathcal{P}_{i, k}$. Investor $i$ 's maximization problem is

$$
\begin{aligned}
\max _{\left(\mathcal{P}_{k, i}, \alpha_{k, i}\right)_{k=1}^{K_{i}}, K_{i}}\{ & \int_{\left(\Sigma \backslash \mathcal{P}_{i}\right) \cap \mathcal{P}_{N}^{*}} \alpha_{i}^{A}\left(\sigma^{2}\right)\left[\pi-\frac{\alpha_{i}^{A}\left(\sigma^{2}\right) \sigma^{2}}{2}\right] d \sigma^{2}+ \\
& \left.+\int_{\left(\Sigma \backslash \mathcal{P}_{i}\right) \cap\left(\Sigma \backslash \mathcal{P}_{N}^{*}\right)} \alpha_{N}^{A}\left(\sigma^{2}\right)\left[\pi-\frac{\alpha_{N}^{A}\left(\sigma^{2}\right) \sigma^{2}}{2}\right] d \sigma^{2}+\sum_{k=1}^{K_{i}} \int_{\mathcal{P}_{i, k}} \alpha_{i, k}\left[\pi-\frac{\alpha_{i, k} \sigma^{2}}{2}\right] d \sigma^{2}\right\} .
\end{aligned}
$$

subject to $K_{i} \in \mathbb{Z}, \mathcal{P}_{k, i} \subseteq \Sigma$ and $\alpha_{i, k} \in[0,1] \forall k=1, . ., K_{i}$.

We start the characterization of the equilibrium with the following lemma.

Lemma 1. In any symmetric equilibrium, neither the pooling region nor the delegation region of the mechanism chosen by the investors are strictly contained in $\Sigma$.

Lemma 1 implies that, once symmetry is imposed, only two types of equilibria with partial delegation may arise: one in which investors pool for low values of $\sigma^{2}$, and delegate otherwise (exactly as in the exclusivity benchmark), the other in which investors delegate for low values of $\sigma^{2}$, and pool otherwise. Of course, there can also exist symmetric equilibria with full pooling or with full delegation, which are however less interesting for our purposes.

\subsection{Portfolio choices perceived as complements by the financial advisor}

Portfolio choices are perceived as complements by the advisor when $\theta<0$. In this case, an increase in $\alpha_{j}$ expands the advisor's ideal portfolio choice of investor $i$ : the more risk one investor takes, the higher the incentive of the advisor to induce the other investor to also take more risk. Because of 
this effect, the common advisor has an incentive to induce each investor to take even more risk than in the case of exclusive advice. Observe that $\alpha_{i}^{A}\left(\sigma^{2}\right) \geq \alpha_{E}^{A}\left(\sigma^{2}\right)$ and $\alpha_{N}^{A}\left(\sigma^{2}\right)>\alpha_{E}^{A}\left(\sigma^{2}\right)$ when $\theta<0$. Hence, the incentives of the financial advisor and those of her clients are even less aligned than under exclusivity. Moreover, the misalignment of incentives exacerbates as $\theta$ decreases. This suggests that under non-exclusive advice investors should trust less the advisor and be less keen to delegate.

Proposition 2. Suppose that $\theta<0$. The game with non-exclusive financial advice has a unique symmetric equilibrium such that:

- If $\theta>(\lambda-\Delta) /(1+\Delta)$, investors partially delegate their investment decisions to the financial advisor and each invests

$$
\alpha_{N}^{*}\left(\sigma^{2}\right)=\left\{\begin{array}{lll}
\alpha_{N}^{*} & \text { if } & \sigma^{2} \leq x_{N}^{*} \\
\alpha_{N}^{A}\left(\sigma^{2}\right) & \text { if } & \sigma^{2}>x_{N}^{*}
\end{array},\right.
$$

with $x_{N}^{*} \equiv \frac{1+\lambda}{1-\lambda+2 \theta}(1-\Delta) \in(1-\Delta, 1+\Delta)$ and $\alpha_{N}^{*}=\frac{\pi}{1-\Delta} \frac{1-\lambda+2 \theta}{1+\theta}$.

- If $\theta \leq(\lambda-\Delta) /(1+\Delta)$, investors ignore the advisor's reports and choose $\alpha_{N}^{*}\left(\sigma^{2}\right)=\pi$ for all $\sigma^{2}$.

Observe that the conditions $\theta<0$ and $\theta>(\lambda-\Delta) /(1+\Delta)$ cannot be satisfied simultaneously when $\lambda>\Delta$. Hence, as in the case of exclusive financial advice, in this region of parameters, investors never delegate the investment decision to the advisor. The reason is that, the conflict of interest that stems from the advisor's intrinsic bias towards an excessive risk exposure is already so strong that delegation is never optimal: full pooling emerges at equilibrium.

However, when $\lambda<\Delta$ delegation emerges in equilibrium as long as the complementarity of portfolio choices is not too strong (i.e., if $\theta$ is not too low). Nevertheless, it is a more limited form of delegation than that in the case of exclusive advice, since $x_{N}^{*}>x_{E}^{*}$. Indeed, as argued above, in this region of parameters the expert's ideal point on each investor is larger with non-exclusive advice than with exclusive advice. Hence, the common advisor has an additional reason to understate the true variance, which makes the investors less keen to trust her and to delegate.

As expected, investors delegate less as $\theta$ decreases. To see why, observe that the condition $\theta>$ $(\lambda-\Delta) /(1+\Delta)$ is less likely to be satisfied for lower values of $\theta$, and that the threshold $x_{N}^{*}$ decreases with $\theta$. In words, a lower $\theta$ makes the advisor more willing to induce excessive risk taking by both investors, which exacerbates the conflict of interest between them and calls for less delegation.

Finally, as in the exclusivity benchmark, delegation becomes more likely as $\Delta$ increases. A larger $\Delta$, which reflects more uncertainty about the state of the world, amplifies the informative advantage of the advisor and thus obliges the investor to rely more often on her. ${ }^{15}$

\footnotetext{
${ }^{15}$ Indeed, the ratio $\frac{x_{N}^{*}-(1-\Delta)}{2 \Delta}=\frac{1-\Delta}{\Delta} \frac{\lambda-\theta}{1-\lambda+2 \theta}$ is decreasing in $\Delta$ also in this case.
} 


\subsection{Portfolio choices perceived as substitutes by the financial advisor}

Portfolio choices are perceived as substitutes by the financial advisor when $\theta>0$. In this region of parameters, an increase in $\alpha_{j}$ reduces the advisor's ideal portfolio choice of investor $i$ : the more risk one investor takes, the lower the incentive of the advisor to induce the other investor to take more risk. As a consequence, the common advisor has an incentive to induce each investor to take less risk than in the case of exclusive financial advice. Observe that $\alpha_{i}^{A}\left(\sigma^{2}\right) \leq \alpha_{E}^{A}\left(\sigma^{2}\right)$ and $\alpha_{N}^{A}\left(\sigma^{2}\right)<\alpha_{E}^{A}\left(\sigma^{2}\right)$ when $\theta>0$. Hence, in this case, non-exclusivity of financial advice mitigates the conflict of interest between the advisor and investors. This suggest that investors should trust more the advisor and delegate more. The following proposition shows that this is true, but only up to a point. If $\theta$ is too high, the advisor may actually prefer that investors invest too little in the risky asset generating a conflict of interest of a different sort.

Proposition 3. Suppose that $\theta>0$. The game with non-exclusive financial advice has a unique symmetric equilibrium. The mechanism chosen by investors in that equilibrium has the following properties:

- If $(\lambda-\Delta) /(1+\Delta)<\theta \leq \lambda$, investors partially delegate their investment decisions to the financial advisor and each invests

$$
\alpha_{N}^{*}\left(\sigma^{2}\right)=\left\{\begin{array}{lll}
\alpha_{N}^{*} & \text { if } \quad \sigma^{2} \leq x_{N}^{*} \\
\alpha_{N}^{A}\left(\sigma^{2}\right) & \text { if } \quad \sigma^{2}>x_{N}^{*}
\end{array}\right. \text {, }
$$

with $x_{N}^{*} \equiv \frac{1+\lambda}{1-\lambda+2 \theta}(1-\Delta) \in(1-\Delta, 1+\Delta)$ and $\alpha_{N}^{*}=\frac{\pi}{1-\Delta} \frac{1-\lambda+2 \theta}{1+\theta}$.

- If $\lambda<\theta<(\lambda+\Delta) /(1-\Delta)$ investors also partially delegate their investment decisions to the financial advisor, but each invests

$$
\alpha_{N}^{*}\left(\sigma^{2}\right)=\left\{\begin{array}{lll}
\alpha_{N}^{A}\left(\sigma^{2}\right) & \text { if } & \sigma^{2} \leq x_{N}^{*} \\
\alpha_{N}^{*} & \text { if } & \sigma^{2}>x_{N}^{*}
\end{array},\right.
$$

with $x_{N}^{*}=\frac{1+\lambda}{1-\lambda+2 \theta}(1+\Delta) \in(1-\Delta, 1+\Delta)$ and $\alpha_{N}^{*}=\frac{\pi}{1+\Delta} \frac{1-\lambda+2 \theta}{1+\theta}$.

- If $\theta \leq(\lambda-\Delta) /(1+\Delta)$ or $\theta \geq(\lambda+\Delta) /(1-\Delta)$ then investors ignore the advisor's reports and choose $\alpha_{N}^{*}\left(\sigma^{2}\right)=\pi$ for all $\sigma^{2}$.

Consider first the case $\theta<\lambda$ - i.e., the advisor perceives the portfolio allocation of her clients as weak substitutes. As expected, delegation is more likely to occur than under exclusivity. ${ }^{16}$ Moreover, investors delegate their portfolio allocation decisions more as $\theta$ increases. Indeed, as $\theta$ increases, either

\footnotetext{
${ }^{16}$ Under exclusive financial advice delegation is optimal only if $\lambda<\Delta$, while here it occurs in equilibrium if $(\lambda-\Delta) /(1+\Delta)<\theta$.
} 
delegation becomes more likely to occur in equilibrium or when it occurs it is of less constrained form $\left(x_{N}^{*}\right.$ decreases with $\left.\theta\right)$. As hinted above, this is because a larger $\theta$ makes the advisor less willing to induce excessive risk taking by both investors, which mitigates the conflict of interest between them and calls for more delegation. Clearly, if more discretion is left to the advisor as a result of a lower conflict of interest, each client is also more eager to invest more into the risky activity because there is less fear of opportunistic behavior by the advisor.

For $\theta=\lambda$ the unique equilibrium of the game with non-exclusive advice trivially yields the first-best outcome (full delegation). Essentially, in this cutting edge case, the negative externality between the portfolio choices stemming from non-exclusive advice exactly compensates the advisor's incentive to induce excessive risk taking, which stems from her intrinsic bias towards an excessive risk exposure.

Consider now the case where $\theta>\lambda$ - i.e., the advisor perceives the portfolio allocation of his clients as strong substitutes. If $\theta$ is not too large, partial delegation may emerge again in equilibrium. However, it is a different form of delegation. Observe that, in this case, the investor sets a minimum on the fraction of his wealth invested into the risky asset. This contrasts with the form of delegation when portfolio choices are weak substitutes, where investors cap the amount invested into the risky asset. The reason is that, with strong substitutability between investment choices, the ideal point of the advisor on each client falls below the first-best level. Hence, the advisor has an incentive to report a variance larger than the true one, so as to induce both clients to take less risk than what they would like to bear. To prevent this type of behavior, in a symmetric equilibrium, it is optimal for both investors to impose a floor on the investment into the risky activity so as to discourage the advisor from over-reporting risk. This leads to a novel type of equilibrium where both investors leave discretion to the advisor when the return volatility of the risky asset is low, and pool when it is sufficiently large.

A graphical illustration of this equilibrium with partial delegation is offered in Figure 2 below:

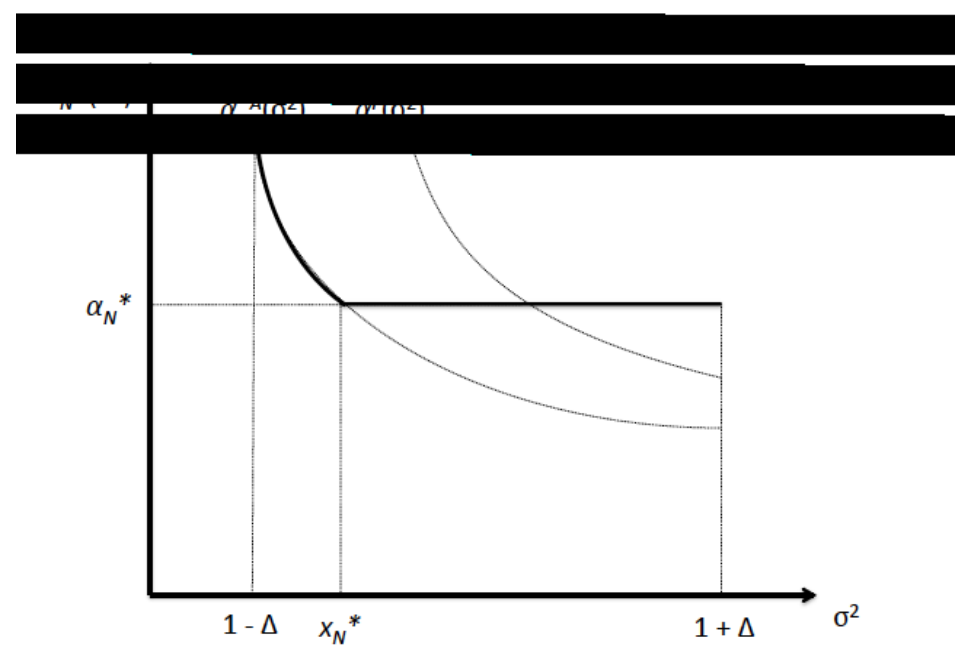

Figure 2: Delegation under non-exclusive financial advice with strong substitutes. 
The equilibrium asset allocation rule is represented by the solid line, which corresponds to the advisor's ideal point for all $\sigma^{2}$ lower than $x_{N}^{*}$, and features a floor for all $\sigma^{2}$ larger than $x_{N}^{*}$.

As in the exclusivity benchmark and the case where portfolio choices are complements, delegation becomes more likely as $\Delta$ increases. However, in contrast to the case where portfolio choices are complements or weak substitutes, when they are strong substitutes the amount of wealth invested into the risky asset within the pooling region $\alpha_{N}^{*}$ is decreasing in $\Delta$ : a larger uncertainty about the state of the world makes investors less willing to risk.

\subsection{Delegated portfolio management and commitment}

Up to this point, we have characterized the equilibrium outcomes of the game by using direct mechanisms. But, are there simpler indirect mechanisms that sustain the portfolio allocations characterized throughout the analysis? Are these indirect mechanisms consistent with real life practices?

It turns out that these indirect mechanisms take an extremely simple form: they require investors to fully delegate their portfolio choices to the advisor, by letting she pick any allocation within a given (compact) set.

Corollary 1. The indirect mechanisms that implement the equilibrium investment choices characterized in Propositions 1, 2 and 3 simply require investors to fully delegate their investment choices to the advisor and bound her choice of risky investment within a given compact set.

Hence, investors do not need to play the communication game analyzed above but, to implement the same equilibrium outcome, they can simply specify the range of investment choices that they are willing to accept, and leave to the advisor full control over the actual portfolio composition: an extremely simple form of delegated portfolio management. Of course, this delegation strategy implicitly assumes that investors commit to accept the investment decision of the financial advisor - i.e., ex post, they commit not to change the portfolio allocation that the advisor has chosen in their behalf. This is the materialization in the context of the application of this indirect mechanism of our hypothesis that investors can commit to the mechanism they use when interacting with the financial advisor.

Interestingly, the existence of disinvestment costs, which reduces investors' incentives to alter investment choices once they have been done, can naturally generate this commitment. Hence, the investor's ability to commit to the mechanism is automatically ensured whenever disinvestment costs are sufficiently large. One important source of disinvestment costs are disinvestment fees. This means that a simple way to solve the commitment problem is to impose sufficiently large disinvestment fees. These fees, which are not paid on the equilibrium path, prevent investors from divesting part of the money allocated by the advisor into a given asset and allocate it into the other asset whenever they manage to infer the state of the world from the advisor's choice. As a result, our equilibrium analysis remains valid 
even under the lack of formal commitment, provided that delegation schemes require disinvestment fees. Actually, these fees, which a priori seem detrimental to investors because they reduce the ability to adjust investment decisions, are welfare enhancing for investors: whenever they are enforceable, disinvestment fees work as a threat that helps eliminate the typical utility loss coming from the lack of commitment. ${ }^{17}$

\section{Investors' expected utility and investment in the risky asset}

Do investors prefer to be in an exclusive relationship with their financial advisors? The answer to this question depends on the relative magnitude of the advisor's bias with and without exclusivity. Intuitively, investors are better off dealing with an exclusive advisor (rather than with an common advisor) if and only if common agency exacerbates the conflict of interests between the investor and the advisor. Formally, this conflict of interest can be measured by the distance between the ideal point of the advisor and the first best allocation - i.e.,

$$
\left|\alpha_{E}^{A}\left(\sigma^{2}\right)-\alpha^{F}\left(\sigma^{2}\right)\right|<\left|\alpha_{N}^{A}\left(\sigma^{2}\right)-\alpha^{F}\left(\sigma^{2}\right)\right| \Leftrightarrow 2 \lambda-\theta(1-\lambda)<0 .
$$

Hence, we can state the following.

Proposition 4. Investors' expected utility is higher with exclusive financial advice than with nonexclusive financial advice when $\theta<0$ or $\theta \geq \frac{2 \lambda}{1-\lambda}>0$. Moreover, the investors' expected utility under non-exclusive financial advice increases with $\theta$ when $\theta<\lambda$, and decreases with $\theta$ when $\theta>\lambda$.

When investment choices are perceived as complements by the advisor, clients exert a negative externality one on the other: with non-exclusive advice, the expert has an extra reason to understate the state of the world and induce excessive risk taking. As a result, the investors' (ex-ante) utility is higher when they deal with an exclusive advisor. Differently, when investment choices are perceived as substitutes by the advisor, clients might exert a positive externality on each other depending on how strong this substitutability is. More precisely, for moderate substitutability - i.e., $0<\theta \leq 2 \lambda /(1-\lambda)$ - the advisor's global incentive to induce excessive risk taking is mitigated under non-exclusive advice via the externality channel: investors generate a positive externality on each other and thus prefer to deal with a common advisor rather than being in an exclusive relationship with her. By contrast, with strong substitutability - i.e., $\theta>2 \lambda /(1-\lambda)$ — the common advisor induces too much under-investment into the risky activity. As a result, investors exert again a negative externality on each other and thus prefer to be in an exclusive relationship with her.

\footnotetext{
${ }^{17}$ On the issue of commitment versus cheap talk see Kolotilin et al. (2013) among others.
} 
Another natural question that emerges from our analysis is whether exclusive financial advice leads investors to invest more (on average) in the risky asset. In order to provide clear cut implications, we will focus on the impact of investors' uncertainty about the return volatility of their investment (as measured by changes in the parameter $\Delta$ ) on the expected investment into the risk activity. To this purpose, let

$$
\hat{\alpha}_{N}^{*} \equiv \int_{1-\Delta}^{1+\Delta} \alpha_{N}^{*}\left(\sigma^{2}\right) \frac{d \sigma^{2}}{2 \Delta}
$$

denote the average investment with non-exclusivity, and

$$
\hat{\alpha}_{E}^{*} \equiv \int_{1-\Delta}^{1+\Delta} \alpha_{E}^{*}\left(\sigma^{2}\right) \frac{d \sigma^{2}}{2 \Delta}
$$

the average investment in the exclusivity benchmark:

Proposition 5. Suppose that $\theta \leq 0$, then $\hat{\alpha}_{N}^{*} \geq \hat{\alpha}_{E}^{*}$ if, and only if, $\Delta$ is large enough. Suppose that $\theta>0$, then $\hat{\alpha}_{N}^{*} \geq \hat{\alpha}_{E}^{*}$ if and only if $\Delta$ is not too large.

This result shows that (ceteris paribus) the impact of non-exclusive advice on the (average) investment into the risky activity depends on the investors' uncertainty about the state of nature. The economic intuition is as follows. Consider first the case of complements $(\theta \leq 0)$. In this region of parameters the advisor's ideal point on each investor is larger with non-exclusivity than with exclusivity (see equation 5.1). This means that (ceteris paribus) when investors delegate, they take more risk when dealing with a common advisor than when dealing with an exclusive advisor, and the opposite holds when they pool. Recall that in both contractual regimes, as $\Delta$ increases there is more reliance on the advisor. Hence, for $\Delta$ large enough, investors dealing with a common advisor invest more (resp. less) into the risky asset because they are more (resp. less) likely to delegate.

Next, consider the case of substitutes $(\theta>0)$. In this region of parameters the advisor's ideal point on each investor is lower with non-exclusivity than with exclusivity (see equation 5.1). This means that (ceteris paribus) when investors delegate, they take less risk when dealing with a common advisor than when dealing with an exclusive advisor. Hence, for $\Delta$ large enough investors dealing with a common advisor invest less (resp. more) into the risky asset because they are more (resp. less) likely to delegate.

\section{Extensions and robustness}

In this section we develop a few natural extensions of the baseline model and perform some robustness checks. 


\subsection{Interpretation of the expert's preferences}

The type of externality investors impose on one another has been treated, so far, as an exogenous feature of the model. In this section we show how the types of externality considered in the baseline analysis may result from the advisor's career concerns, the structure of the sales commissions paid by the provider of the financial product and the investors' ex post decisions to fire the advisor. In other words, we show how these ingredients (un-modeled so far) may originate an interaction between the clients' investment choices in the advisor's utility function. Clearly, while in equation (3.3) we have assumed, for tractability, that this interaction is linear, in reality it can be more complex (i.e., nonlinear), so that the forces that drive complementarity and substitutability may be simultaneously at work. To simplify exposition, here we will consider each case in isolation.

Complementarity. A simple way to generate complementarity between the investors' portfolio choices in the expert's objective function is to assume that she is exposed to sales commissions that are convex in the volume of sales. The idea that sales incentives create an agency conflict, which harms investors, is not new in the literature. For example, Ottaviani (2000) shows that the intrinsic bias $\lambda$ can be interpreted as the result of linear commission fees. However, recent anecdotal evidence offered by two reports, conducted respectively by the Financial Services Authority (FSA) and by Consumers International, highlights several instances in which 'convex' fees can significantly increase the risk of misselling of financial products. ${ }^{18}$ The practices described in these reports include, among others:

- Accelerators, stepped payments and thresholds: Once advisors achieve a certain 'threshold' (a minimum level of sales), they are given 'higher rates of incentives' on each additional sale.

- Disproportionate rewards for marginal sales: Reaching a certain target or a goal that triggers an increase in earnings much higher than the normal rate at which incentives accrue. An example is a 'retrospective accelerator' where passing a target increases the level of incentive earned for all sales over a period, rather than just those above the target.

To illustrate how a convex commission scheme may lead an advisor to perceive her clients' portfolio choices as complements, let $C\left(\alpha_{1}+\alpha_{2}\right)$ denote the commission scheme as a function of the total sales $\alpha_{1}+\alpha_{2}$, with $C(\cdot)$ being twice continuously differentiable, $C^{\prime}(\cdot) \geq 0, C^{\prime \prime}(\cdot) \geq 0$ and $C(0)=C^{\prime}(0)=0 .{ }^{19}$ Suppose, just for simplicity, that these commissions are entirely passed through by the bank to the (riskneutral) advisor. Finally, following Ottaviani (2000), suppose the expert's expected utility is equal to

\footnotetext{
${ }^{18}$ These reports are available at:

https://www.fca.org.uk/static/fca/documents/finalised-guidance/fsa-fg13-01.pdf

http://www.consumersinternational.org/media/1529404/sales-incentive-report_riskybusiness_final2_151014.pdf

${ }^{19}$ The assumption that $C(\cdot)$ is a twice continuously differentiable function is just a convenient approximation.
} 
the sum of her clients' expected utility plus the commissions. In this case, the expert's expected utility is given by

$$
v\left(\boldsymbol{\alpha}, \sigma^{2}\right)=\sum_{i=1,2}\left[\alpha_{i}\left(\mu-r_{f}\right)+r_{f}-\frac{\gamma}{2} \alpha_{i}^{2} \sigma^{2}\right]+C\left(\alpha_{1}+\alpha_{2}\right)
$$

Under a standard concavity requirement ${ }^{20}$, the advisor's ideal level of investment in the risky asset by investor $i$ (given the investment level $\alpha_{j}$ by investor $j$ ), in an internal solution, solves the first-order condition

$$
\mu-r_{f}-\gamma \alpha_{i} \sigma^{2}+C^{\prime}\left(\alpha_{i}+\alpha_{j}\right)=0
$$

which implies that $\alpha_{i}$ and $\alpha_{j}$ are strategic complements - i.e., using the Implicit Function Theorem

$$
\frac{\partial \alpha_{i}}{\partial \alpha_{j}}=\frac{C^{\prime \prime}\left(\alpha_{i}+\alpha_{j}\right)}{\left|\gamma \sigma^{2}-C^{\prime \prime}\left(\alpha_{i}+\alpha_{j}\right)\right|}>0
$$

Hence, the advisor prefers an investor to allocate a larger fraction of his wealth into the risky activity when the other investor does so too. This complementarity becomes stronger when the compensation scheme becomes relatively more convex - i.e., when (other things being equal) the function $C^{\prime \prime}(\cdot)$ increases.

Substitutability. Explaining substitutability is less straightforward. One obvious way to do it, which is immediately suggested by (7.1), would be to assume that sales commissions are concave rather than convex - i.e., that $C^{\prime \prime}(\cdot)<0$. However, while convexity of these compensation schemes is plausible as argued above, concavity seems harder to justify, unless binding regulatory constraints impose a cap on these payments, which tends to make them concave. ${ }^{21}$ Yet, the following are two situations in which substitutability is likely to emerge in the advisor's utility function.

Advisor with career concerns. Suppose that, in addition to sales incentives (which are assumed linear for simplicity), the advisor's 'wealth' $w$ depends on the performance of her portfolio, perhaps because her career advances depend on performance - i.e.,

$$
w(\tilde{r}, \boldsymbol{\alpha})=\underbrace{\left(\tilde{r}-r_{f}\right) \sum_{i=1,2} \alpha_{i}}_{\text {Career concerns }}+\underbrace{\tau \sum_{i=1,2} \alpha_{i}}_{\text {Sales incentives }} .
$$

Suppose also the advisor is risk averse with a mean-variance expected utility, whose risk aversion

\footnotetext{
${ }^{20}$ That is, the advisor's expected utility is concave along each dimensions $\alpha_{i}$, which in our example requires $C^{\prime \prime}(\cdot)$ not too positive.

${ }^{21}$ Actually, the reports discussed above advocate in favour of such regulations in order to reduce the risk of misselling of financial products.
} 
coefficient is $\rho$. Using condition (7.2), her expected utility in this case can be written as

$v\left(\boldsymbol{\alpha}, \sigma^{2}\right)=\left(\mu-r_{f}+\tau\right) \sum_{i=1,2} \alpha_{i}-\frac{\rho}{2}\left[\sum_{i=1,2} \alpha_{i}\right]^{2} \mathbb{E}[\tilde{r}-\mu]^{2}=\sum_{i=1,2}\left[\left(\mu-r_{f}+\tau\right) \alpha_{i}-\frac{\rho}{2} \sigma^{2} \alpha_{i}^{2}\right]-\underbrace{\rho \alpha_{1} \alpha_{2} \sigma^{2}}_{\text {Interaction term }}$.

Hence, for given $\sigma^{2}$, substitutability is captured by the advisor's risk aversion coefficient $\rho$. In fact, in order to edge the income risk, the expert tends to be more prudent when giving advice to one client if she has induced the other client to take enough risk.

Advisor who faces firing costs. Consider now a situation in which, once the return of the risky asset is realized, investors may decide to change advisor if they perceive that the investment has not performed sufficiently well. More formally, suppose investor $i$ 'fires' the advisor if, and only if,

$$
\alpha_{i}\left(\tilde{r}-r_{f}\right) \leq \tilde{z}_{i}
$$

where $\tilde{z}_{i}$ is a random variable (i.i.d. across investors and independent of the stochastic return $\tilde{r}$ ) distributed according to a continuous c.d.f. $H\left(z_{i}\right)$ with associated p.d.f. $h\left(z_{i}\right)$. The variable $\tilde{z}_{i}$ can be interpreted as investor $i$ 's unanticipated 'irritational emotions' - i.e., all those factors, exogenous to the relationship between the investor and the advisor, that may induce the former to fire the latter. ${ }^{22}$ The larger the value of $\tilde{z}_{i}$ the more likely is that the advisor will be fired by investor $i$. A similar 'idea' is developed in Kaniel and Kondor (2013), who also discuss anecdotal evidence suggesting that the capital at the disposal of top traders at investment banks and hedge funds is positively correlated with their past performance. Firing decisions are, in fact, an extreme form of 'irrational behavior' that may induce investors to cut down the amount invested in a risky activity after a bad performance.

Hence, for any risky investment $\alpha_{i}$, the probability that investor $i$ fires the advisor conditional on a given return $\tilde{r}$ is

$$
1-\operatorname{Pr}\left[\tilde{z}_{i} \leq \alpha_{i}\left(\tilde{r}-r_{f}\right) \mid \tilde{r}\right]=1-H\left(\alpha_{i}\left(\tilde{r}-r_{f}\right)\right)
$$

To make our point in the simplest possible way, we assume (without loss of insights) that the advisor's (anticipated) cost of being fired is positive and equal to $\beta$ only when both clients quit and

\footnotetext{
${ }^{22}$ Extensive evidence from psychology and empirical finance suggests that people place greater weight on information gained through personal experience. First described by Tversky and Kahneman (1974) as availability bias, this idea has gained considerable support in recent empirical literature. For example, Malmendier and Nagel (2011) show that the predictions by investors who have experienced low stock market returns throughout their lives are more cautious.
} 
zero otherwise. ${ }^{23,24}$ Conditional on $\tilde{r}$, this happens with probability

$$
\left[1-H\left(\alpha_{1}\left(\tilde{r}-r_{f}\right)\right)\right] \times\left[1-H\left(\alpha_{2}\left(\tilde{r}-r_{f}\right)\right)\right]
$$

The expected (unconditional) cost for the advisor of being fired is

$$
\beta \int_{\tilde{r}}\left[1-H\left(\alpha_{1}\left(\tilde{r}-r_{f}\right)\right)\right] \times\left[1-H\left(\alpha_{2}\left(\tilde{r}-r_{f}\right)\right)\right] d G(\tilde{r}),
$$

where $G($.$) is the c.d.f. of \tilde{r}$. Assuming, as before, that the expert cares about the sum of the clients' expected utility and the (linear) sales commissions, her unconditional expected utility net of the firing cost is given by

$$
v\left(\boldsymbol{\alpha}, \sigma^{2}\right)=\sum_{i=1}^{2}\left[r_{f}+\alpha_{i}\left(\mu-r_{f}+\tau\right)-\frac{\gamma}{2} \alpha_{i}^{2} \sigma^{2}\right]-\beta \int_{\tilde{r}} \prod_{i=1,2}\left[1-H\left(\alpha_{i}\left(\tilde{r}-r_{f}\right)\right)\right] d G(\tilde{r})
$$

Maximizing $v(\cdot)$ with respect to $\alpha_{i}$ yields, in the case of an internal solution, the first-order condition

$$
\mu-r_{f}+\tau-\gamma \alpha_{i} \sigma^{2}+\beta \int_{\tilde{r}}\left(\tilde{r}-r_{f}\right) h\left(\alpha_{i}\left(\tilde{r}-r_{f}\right)\right)\left[1-H\left(\alpha_{j}\left(\tilde{r}-r_{f}\right)\right)\right] d G(\tilde{r})=0,
$$

which, under a standard concavity requirement ${ }^{25}$, implies that $\alpha_{i}$ and $\alpha_{j}$ are substitutable - i.e., using the Implicit Function Theorem

$$
\frac{\partial \alpha_{i}}{\partial \alpha_{j}}=-\frac{\beta \int_{\tilde{r}}\left(\tilde{r}-r_{f}\right)^{2} h\left(\alpha_{i}\left(\tilde{r}-r_{f}\right)\right) h\left(\alpha_{j}\left(\tilde{r}-r_{f}\right)\right) d G(\tilde{r})}{\left|\gamma \sigma^{2}-\beta \int_{\tilde{r}}\left(\tilde{r}-r_{f}\right)^{2} h^{\prime}\left(\alpha_{i}\left(\tilde{r}-r_{f}\right)\right)\left[1-H\left(\alpha_{j}\left(\tilde{r}-r_{f}\right)\right)\right] d G(\tilde{r})\right|}<0 .
$$

Substitutability here results from the need of the advisor to hedge the risk of being fired by both clients. In fact, the higher is $\beta$, the stronger the substitutability between $\alpha_{i}$ and $\alpha_{j}$ (other things being equal). Noteworthy, the parameter $\beta$ can be interpreted as a proxy of the cost of reputation that the advisor bears when both her clients quit. A higher $\beta$ may reflect, for instance, a higher distance from retirement - i.e., older advisors should care less about their reputation. In this perspective, our analysis suggests that older advisors, whose $\beta$ should be relatively low, should induce (other things being equal) their clients to take excessive risk in order to cash commission fees. This prediction is consistent with the evidence provided by Foerster et al. (2014), who find that older advisors are more willing to give their clients riskier products because, being close to or over the retirement age, they do not need to be

\footnotetext{
${ }^{23}$ The advisor may anticipate the investor's firing behavior due to her past experience or insider knowledge of the market.

${ }^{24}$ When the advisor bears a reputational cost even when only one client fires her, the result of the analysis does not change qualitatively provided that the cost of loosing both clients is sufficiently larger than the cost of loosing only one.

${ }^{25}$ That is, the advisor's expected utility is concave along each dimension $\alpha_{i}$, which in our example requires $h^{\prime}(\cdot)$ not too positive.
} 
as concerned about any detrimental effect that this behavior might have on their reputation. On the contrary, younger advisors seem to hold a more prudent behavior.

\subsection{Multiple clients}

In this section we show that the structure of the equilibrium investment rules does not change (qualitatively) when, instead of two clients, the advisor has $N>2$ clients. Clearly, regardless of the number of clients, whether the advisor perceives their investment choices as complements or as substitutes is still key to determine the equilibrium of the game. Hence, the basic effects of non-exclusive financial advice and the structure of the investments rule obtained in the baseline model extend, qualitatively, to the case where the advisor serves several clients. The interesting aspect to address here is, therefore, how the misalignment of preferences between the expert and each investor varies when the number of her clients increases. In what follows we show that, at least in some cases, this misalignment becomes more pronounced as the number of clients increases, which (as in the analysis developed above) induces less delegation in equilibrium.

Before detailing the formal arguments, there is one important consideration the reader should be aware of. Specifically, there are several arbitrary ways to introduce multiple investors into the loss function (3.3), each delivering potentially different predictions. As explained before, this is because the loss-function in (3.3) is just a simplifying and quite flexible analytical tool, which allows us to capture complementarity and substitutability in a unified framework. Hence, to avoid the ambiguity of making claims based on a reduced form approach, we will study the impact of the number of investors within the context of the examples developed in Section 7.1.

Consider first the case of complementarity. Suppose (just for simplicity) that the compensation scheme $C(\cdot)$ is quadratic - i.e.,

$$
C(\cdot)=\underbrace{\tau \sum_{i=1}^{N} \alpha_{i}}_{\text {Linear component }}+\underbrace{\eta\left[\sum_{i=1}^{N} \alpha_{i}\right]^{2}}_{\text {Convex component }},
$$

where $N$ is the number of clients the advisor serves. Following the approach used in the previous sections, we obtain that the advisor's (symmetric) ideal point is

$$
\alpha_{N}^{A}\left(\sigma^{2}\right)=\frac{\mu-r_{f}+\tau}{\gamma \sigma^{2}-2 N \eta}
$$

which is increasing in $N$. In fact, because investment choices are complements, a larger number of clients would make the advisor even more willing to induce excessive risk in order to exploit convexity of her compensation scheme - i.e., it would exacerbate the conflict of interest between the advisor and 
her clients, leading to even less delegation in a symmetric equilibrium.

Consider now the case of substitutability. For brevity, we focus only on the more tractable case of career concerns. With $N$ clients the advisor's future wealth is

$$
w(\cdot)=\left(\tilde{r}-r_{f}\right) \sum_{i=1}^{N} \alpha_{i} .
$$

Hence, her (symmetric) ideal point is

$$
\alpha_{N}^{A}\left(\sigma^{2}\right)=\frac{\mu-r_{f}}{\rho N \sigma^{2}}
$$

which is decreasing in $N$. This implies that, even with substitutability, everything else equal, a larger pool of clients exacerbates the conflict of interest between the advisor and her clients, inducing the advisor to behave more prudently than in the baseline model.

To conclude, in both cases, as the number of clients increases, the conflict of interest between the advisor and her clients becomes more pronounced. Hence, in equilibrium, investors delegate less, which means that the pooling region expands. Of course, this conclusion should be interpreted with care. The examples studied above highlight only two of many alternative ways in which the investors' choices may interact in the advisor's utility function. In practice, other forces may be simultaneously at work, and encompassing all in a tractable model may be a very complex task, as they may work in opposite directions. Hence, the assessment of the impact of $N$ on the conflict of interest between investors and their common advisor seems more of an empirical matter, which surprisingly has not been studied yet.

\subsection{Heterogeneous investors}

In this section we allow investors to differ in the information they own and in their degree of risk aversion. For comparison purposes, we will return to the reduced form approach of Section 3.

Investors with different information about $\sigma^{2}$. Suppose that investors have different information about $\sigma^{2}$, although they feature the same risk aversion coefficient. For simplicity, assume that investor 1 is perfectly informed about the realization of the state of the world and thus chooses the first best allocation, whereas investor 2 is uncertain about the realization of $\sigma^{2}$ and (as before) has a uniform prior distributed over the support $\Sigma$. We could think of investor 1 as being a very sophisticated investor. A higher (resp. lower) $\Delta$ can be interpreted as a larger (resp. smaller) heterogeneity between investors.

Since investor 1 is perfectly informed about the state of the world, only investor 2 has to elicit truthful information revelation from the advisor. Incentive compatibility requires $\alpha_{2}\left(\sigma^{2}\right)$ is either flat or if it is equal to the advisor's ideal point

$$
\alpha_{2}^{A}\left(\sigma^{2}\right)=(1+\lambda-\theta) \alpha^{F}\left(\sigma^{2}\right)
$$


Observe that the difference between the advisor's ideal point and the investor's ideal point depends only on the sign of $\lambda-\theta$ and that

$$
\alpha_{2}^{A}\left(\sigma^{2}\right)-\alpha^{F}\left(\sigma^{2}\right)=(\lambda-\theta) \alpha^{F}\left(\sigma^{2}\right) \geq 0 \quad \Leftrightarrow \quad \lambda \geq \theta
$$

Condition (7.3) suggests that the forces at play in this simple asymmetric environment are similar to those described in the case of symmetric investors. Specifically, when the advisor perceives the investment choices of her clients as complements $(\theta \leq 0)$ or as weak substitutes $(0<\theta<\lambda)$, she has an incentive to induce the uninformed investor to take excessive risk. Hence, the portion of wealth that investor 2 allocates to the risky activity needs to be capped. By contrast, when investment choices are perceived as strong substitutes $(\lambda<\theta)$, the advisor has an incentive to induce the uninformed client to underinvest into the risky asset, which in turn requires the portion of wealth allocated to the risky activity to be floored. Clearly, for $\lambda=\theta$ the first-best allocation is implemented.

Relative to the case where investors are symmetric, the conflict of interest between the advisor and his uninformed client is amplified: the advisor cannot manipulate the choice of the fully informed investor 1 , so the cost of truthful information revelation has to be paid only by the uninformed investor 2. This suggests that less informed investors are those who need to distort more their financial choices when dealing with a common advisor and are those who benefit more from exclusive financial advice.

Investors with different risk attitudes. Consider now investors that differ in the degree of risk aversion, but have the same information. For simplicity, consider the case where investor 1 is risk neutral, so that his wealth is entirely invested into the risky asset regardless of the advisor's report $m_{1}$, while investor 2's risk averse coefficient is $\gamma>0$. Hence, a higher $\gamma$ (resp. lower) captures a larger (resp. smaller) asymmetry between the investors.

Since investor 1 is risk neutral, only investor 2 has to elicit a truthful report from the advisor. Using the same techniques developed in Section 5, it is easy to verify that incentive compatibility requires that $\alpha_{2}\left(\sigma^{2}\right)$ is either unresponsive to $\sigma^{2}$ or if it is equal to the advisor's ideal point

$$
\alpha_{2}^{A}\left(\sigma^{2}\right)=(1+\lambda) \alpha^{F}\left(\sigma^{2}\right)-\theta
$$

In contrast to the results stated in the previous sections, with different attitudes towards risk new interesting outcomes may arise. To see why, it is useful to compare the advisor's ideal point with the first-best rule - i.e.,

$$
\alpha_{2}^{A}\left(\sigma^{2}\right)=(1+\lambda) \alpha^{F}\left(\sigma^{2}\right)-\theta \geq \alpha^{F}\left(\sigma^{2}\right) \Leftrightarrow \frac{\pi}{\sigma^{2}} \geq \frac{\theta}{\lambda} .
$$


Hence, for $\theta \leq 0$ or $\theta>0$ but not too large, the advisor's ideal point always exceeds the first best choice. This suggests that when the advisor perceives the clients' investment allocation choices as complements - i.e., $\theta \leq 0$ - or as weak substitutes - i.e., $\theta<\lambda \alpha^{F}\left(\sigma^{2}\right)$ for every $\sigma^{2}$ - investor 2's optimal delegation strategy still requires to cap the portion of wealth invested into the risk asset. When, instead, asset allocations are perceived as very strong substitutes by the advisor - i.e., $\theta>\lambda \alpha^{F}\left(\sigma^{2}\right)$ for every $\sigma^{2}$ - the advisor's ideal point falls short of the first-best choice. In this case, investor 2's optimal delegation strategy requires a floor on the amount invested into the risky asset. However, with heterogenous attitude towards risk, a novel interesting outcome emerges. This case occurs when there exists a $\hat{\sigma}^{2} \in(1-\Delta, 1+\Delta)$ such that $\lambda \alpha^{F}\left(\hat{\sigma}^{2}\right)=\theta-$ i.e.,

$$
1+\Delta>\hat{\sigma}^{2} \equiv \frac{\lambda \pi}{\theta}>1-\Delta
$$

At $\sigma^{2}=\hat{\sigma}^{2}$ the investor's ideal point coincides with the first best. Hence, $\alpha_{2}^{A}\left(\sigma^{2}\right)>\alpha^{F}\left(\sigma^{2}\right)$ for values of $\sigma^{2}$ lower than $\hat{\sigma}^{2}$ : in this region of parameters, investor 2 would like to cap the portion of wealth that he invests into the risky asset. By contrast, $\alpha_{2}^{A}\left(\sigma^{2}\right)<\alpha^{F}\left(\sigma^{2}\right)$ for values of $\sigma^{2}$ that exceed $\hat{\sigma}^{2}$ : in this region of parameters investor 2's optimal investment choice is to impose a floor on the amount of wealth that he can invest into the risky asset.

While the first two types of behavior have already been discussed in the case of symmetric investors and heterogenous beliefs, the third one is novel and hinges only on the hypothesis that investors are heterogeneous with respect to their risk attitude. In this case, investor 2's optimal delegation strategy requires both a cap and a floor. Since $\pi$ is decreasing in $\gamma$, this suggests that an optimal delegation strategy requires both a cap and a floor only for people with moderate risk aversion, who tend to rely less often on financial advice when buying assets with extremely low or excessively large return volatility. The reason is that very risk averse people tend to impose only a cap on the amount of wealth they are willing to invest into the risky asset because their ideal investment choice entails most likely less risk than what the advisor would like to induce; by contrast, less risk averse people tend to impose only a floor on the amount of wealth they are willing to invest into the risky asset because their ideal investment choice entails most likely more risk than what the advisor would like to induce. Hence, moderate risk averse people may induce both a cap and a floor. ${ }^{26}$

\footnotetext{
${ }^{26}$ Of course, to simplify our life, in this section we have taken the extreme case in which one investor is completely risk neutral while the other is risk-averse. What would happen if they were both risk averse? Of course, if there is a negligible difference in their attitude towards risk, all the qualitative results found in the baseline model continue to hold here too by continuity. Otherwise, one should expected the following. With complements or weak substitutes, the conflict of interest between the advisor and his clients is largest for the more risk averse investor, who is the one that is likely to impose a tighter cap on his equilibrium investment decision. By contrast, with strong substitute, the conflict of interest is stronger with the less risk averse investor, who is the one that is likely to impose a tighter floor in equilibrium.
} 


\subsection{Multidimensional asymmetric information: basic insights}

The analysis developed so far hinges on the assumption that investors know $\lambda$ and $\theta$. Yet, while it seems reasonable to assume that clients know the advisor's intrinsic bias $\lambda$ (a standard assumption in the cheap talk literature), which in many models reflects the linear component of the advisor's commission fees (see, e.g., Ottaviani, 2000), they may not know how the advice they receive is affected by the presence of the other client - i.e., each client may have only imperfect information on $\theta$ or, alternatively, be uncertain about the presence of the other client. ${ }^{27}$ Hence, a natural question is whether our results remain qualitatively valid when the advisor is better informed than the investors not only on $\sigma^{2}$ but also on $\theta$ and, therefore, is asked to report both.

In this section we partially address this issue by studying the basic properties of the equilibrium mechanisms with two dimensions of asymmetric information. ${ }^{28}$ Suppose investors believe $\theta$ is distributed according to some continuously differentiable c.d.f. in the support $\Theta$. Each investor $i$ chooses a direct mechanism $\mathcal{M}_{i} \equiv\left\{\alpha_{i}\left(m_{i}, t_{i}\right)\right\}_{m_{i} \in \Sigma, t_{i} \in \Theta}$, with $\alpha_{i}(\cdot): \Sigma \times \Theta \rightarrow[0,1]$, which specifies a portfolio allocation $\alpha_{i}\left(m_{i}, t_{i}\right)$ for any (private) pair of reports $m_{i} \in \Sigma$ and $t_{i} \in \Theta$, made by the advisor to investor $i$ about the state of nature $\left(\sigma^{2}, \theta\right)$. As before, we focus on continuous and piecewise differentiable mechanisms.

Given mechanisms $\alpha_{1}(\cdot)$ and $\alpha_{2}(\cdot)$, the advisor' utility is

$$
v\left(\alpha_{1}\left(m_{1}, t_{1}\right), \alpha_{2}\left(m_{2}, t_{2}\right), \sigma^{2}, \theta\right)=-\frac{1}{2} \sum_{i=1,2}\left[\alpha_{i}\left(m_{i}, t_{i}\right)-(1+\lambda) \alpha^{F}\left(\sigma^{2}\right)\right]^{2}-\theta \alpha_{1}\left(m_{1}, t_{1}\right) \alpha_{2}\left(m_{2}, t_{2}\right),
$$

if she reports $\left(m_{1}, t_{1}\right)$ and $\left(m_{2}, t_{2}\right)$ when the true state of nature is $\left(\sigma^{2}, \theta\right)$.

Using the same logic as before, the (local) incentive compatibility conditions, necessary to guarantee truthful information revelation are

$$
\begin{aligned}
& \frac{\partial}{\partial m_{i}} v\left(\alpha_{1}\left(\sigma^{2}, \theta\right), \alpha_{2}\left(\sigma^{2}, \theta\right), \sigma^{2}, \theta\right)=0 \Leftrightarrow\left[\alpha_{i}\left(\sigma^{2}, \theta\right)-(1+\lambda) \alpha^{F}\left(\sigma^{2}\right)+\theta \alpha_{j}\left(\sigma^{2}, \theta\right)\right] \frac{\partial \alpha_{i}\left(\sigma^{2}, \theta\right)}{\partial m_{i}}=0, \\
& \frac{\partial}{\partial t_{i}} v\left(\alpha_{1}\left(\sigma^{2}, \theta\right), \alpha_{2}\left(\sigma^{2}, \theta\right), \sigma^{2}, \theta\right)=0 \Leftrightarrow\left[\alpha_{i}\left(\sigma^{2}, \theta\right)-(1+\lambda) \alpha^{F}\left(\sigma^{2}\right)+\theta \alpha_{j}\left(\sigma^{2}, \theta\right)\right] \frac{\partial \alpha_{i}\left(\sigma^{2}, \theta\right)}{\partial t_{i}}=0,
\end{aligned}
$$

for every $i=1,2$. Observe that the first terms of these conditions are identical, which implies that an incentive compatible mechanism is either flat along both dimensions of asymmetric information — i.e., $\partial \alpha_{i}\left(\sigma^{2}, \theta\right) / \partial m_{i}=\partial \alpha_{i}\left(\sigma^{2}, \theta\right) / \partial t_{i}=0$ - or it requires

$$
\alpha_{i}\left(\sigma^{2}, \theta\right)=(1+\lambda) \alpha^{F}\left(\sigma^{2}\right)-\theta \alpha_{j}\left(\sigma^{2}, \theta\right)
$$

\footnotetext{
${ }^{27}$ As the reader will realize in what follows, the absence of monetary transfers makes the multidimensional screening problem quite easy to account also for uncertainty about $\lambda$, while still preserving the main qualitatively features of the baseline results.

${ }^{28}$ For brevity, we do not address here the technical issues related to the existence of equilibrium. The objective of this section is solely to show that even when $\theta$ is unknown, the investment choices feature properties that are qualitatively similar to those analyzed in the main model.
} 
Imposing symmetry, this condition yields again

$$
\alpha_{N}^{A}\left(\sigma^{2}, \theta\right)=\frac{(1+\lambda) \alpha^{F}\left(\sigma^{2}\right)}{1+\theta}
$$

As a result, within the type space $\Sigma \times \Theta$ the equilibrium outcome is simple: it entails an investment choice that is either unresponsive to $\sigma^{2}$ and $\theta$ or it implements the advisor's ideal point $\alpha_{N}^{A}\left(\sigma^{2}, \theta\right)$. Indeed, note that

$$
\alpha_{N}^{A}\left(\sigma^{2}, \theta\right)>\alpha^{F}\left(\sigma^{2}\right) \quad \Leftrightarrow \quad \lambda>\theta
$$

which implies that for values of $\theta$ relatively small, the equilibrium should feature a cap when $\sigma^{2}$ is small enough, whereas for values of $\theta$ large the equilibrium should feature a floor when $\sigma^{2}$ is large enough. Hence, assuming away corner solutions in which only one dimension of asymmetric information matters ${ }^{29}$, an equilibrium with partial delegation (if it exists) entails a pooling outcome when $\sigma^{2}$ and $\theta$ are both small (cap) and when they are both large (floor), while it features separating when $\sigma^{2}$ and $\theta$ take intermediate values.

\section{Concluding remarks}

When investors deal with a common financial advisor, there may exist externalities between investors that arise from the expert's preference structure. These externalities affect equilibrium asset allocation choices in a non-obvious way. To highlight this point and its implications for investment behavior and welfare, we have developed a stylized model of non-exclusive financial advice in which investors rely on a common expert to make their portfolio choices. Although it is difficult to validate our results with the available evidence, because up to now there has been no attempt to tackle empirically the impact of non-exclusive advice financial markets, our results advance the current understanding of how clients and advisors are expected to behave in a common agency setting where monetary transfers are not allowed, and offer new ground for future empirical investigations. Specifically, the analysis has delivered the following testable implications on the effect of non-exclusivity of financial advice on investment behavior.

First, relative to earlier models in which financial advice is exclusive, in our setting when portfolio choices are more likely to be perceived as complements or strong substitutes investors should trust less the expert and be less keen to delegate. By contrast, when portfolio choices are more likely to be perceived as weak substitutes investors should trust more the expert and be more keen to delegate relative to the exclusivity benchmark. Evidence pointing in this direction is found in Monti et al. (2014) who investigate factors that influence trust and advice, finding empirical evidence supporting the idea

\footnotetext{
${ }^{29}$ Intuitively, for this to be true it is suffcient that there is enough variation in both states - i.e., that the length of the intervals $\Theta$ and $\Sigma$ is not too asymmetric and that $\lambda \in \operatorname{int} \Theta$.
} 
that people tend to trust more advisors that they perceive as being relatively less biased.

Second, the model predicts that depending on whether portfolio choices are perceived as strong substitutes or weak substitutes/complements, the optimal investment strategy requires a floor or a cap on the amount of wealth invested into the risky asset, respectively. As explained before - see Figures 1 and 2 - this has interesting empirical implications for actual portfolio choices. In fact, when the optimal investment strategy requires a cap (e.g., when experts are exposed to convex sales incentives), investors are relatively more likely to delegate when buying products with a high risk profile. By contrast, when it is optimal to impose a floor (e.g., when experts care particularly about reputation), investor are more likely to delegate when buying products with a low risk profile. Although there is no available evidence on the correlation between investor's propensity to delegate and the characteristics of the products they buy, the emergence of an equilibrium with partial delegation, whose features do not depend exclusively on investors' characteristics, seems consistent with the evidence collected by Foerster et al. (2014) who find that clients' observable characteristics jointly explain only $11 \%$ of the variation in risky share in the cross-section of Canadian households. They argue that advisor fixed effects have substantial explanatory power and impute this evidence to the advisors' idiosyncratic 'tastes' in portfolio allocation, which may reflect agency conflicts similar to those highlighted in our paper.

Third, the model confirms the pervasive perception in the industry that 'convex' sales incentives (such as accelerators, stepped payments, thresholds and so on) exacerbate the incentive to induce excessive risk taking, especially when financial advice is non-exclusive, and it also implies that reputation concerns may mitigate this incentive - i.e., older advisors, whose cost of being fired is relatively small, should induce their clients to take excessive risk in order to cash larger commission fees. This latter prediction is consistent with the evidence provided by Foerster et al. (2014), who find that older advisors are more willing to give their clients riskier products because, being close to or over the retirement age, they do not need to be as concerned about any detrimental effect that this behavior might have on their reputation. On the contrary, younger advisors seem to hold a more prudent behavior.

Fourth, when accounting for more than two clients, it seems that the conflict of interest between the advisor and each individual investor exacerbates as the number of clients grows larger (at least in some cases of interest). Finally, when accounting for asymmetries between investors, the analysis implies that: less informed investors are those who need to distort more their financial choices when dealing with a common advisor and are those who benefit more from exclusive financial advice; asymmetries in risk aversion may have instead strong implications on the type of strategy investors enact. Specifically, people that are not too risk averse tend to delegate more when buying assets with a high risk profile, people with moderate risk aversion delegate more when they buy assets with intermediate risk, while very risk averse people delegate only when buying assets with a low risk profile.

Taken together, these predictions imply that non-exclusivity in financial advice may matter sub- 
stantially not only to understand people investment behavior, but also to predict the potential welfare effects of policies that regulate (or, at least, impact) the structure of the commission fees paid to retail banks by the providers of financial products, the nature of banks' internal sales incentives and the advisor's career prospects. Hence, this specific dimension seems to deserve more close attention by the empirical research. 


\section{A. Appendix}

Proof of Proposition 1. We begin by showing that if the optimal mechanism $\mathcal{M}_{E}^{*}$ is such that the investor pools in a subset $\mathcal{P}$ of $\Sigma$, then $\mathcal{P}$ cannot be strictly contained in $\Sigma$.

Suppose that the investor pools only in the subset $\mathcal{P} \equiv[x, y] \subset \Sigma$, with $1-\Delta<x<y<1+\Delta-$ i.e., he invests a share $\alpha$ of his wealth into the risky asset for every $\sigma^{2} \in \mathcal{P}$. Then, incentive compatibility requires an investment $\alpha_{E}^{A}\left(\sigma^{2}\right)$ for every $\sigma^{2} \in \Sigma \backslash \mathcal{P}$. The investor's maximization problem is

$$
\max _{\mathcal{P}, \alpha} \mathcal{W}(\mathcal{P}, \alpha) \equiv \max _{x, y, \alpha}\left\{\int_{x}^{y} \alpha\left[\pi-\frac{\alpha \sigma^{2}}{2}\right] d \sigma^{2}+\int_{\Sigma \backslash[x, y]} \alpha_{E}^{A}\left(\sigma^{2}\right)\left[\pi-\frac{\alpha_{E}^{A}\left(\sigma^{2}\right) \sigma^{2}}{2}\right] d \sigma^{2}\right\}
$$

subject to $\mathcal{P} \subset \Sigma$ and $\alpha \in[0,1]$.

The first-order condition with respect to $\alpha$ is

$$
\alpha=\frac{\pi}{\mathbb{E}\left[\sigma^{2} \mid \sigma^{2} \in \mathcal{P}\right]} \equiv \frac{2 \pi}{x+y} .
$$

Continuity of the mechanism $\mathcal{M}$ implies $\alpha_{E}^{A}(x)=\alpha=\alpha_{E}^{A}(y)$. Using (.1) and $\alpha_{E}^{A}\left(\sigma^{2}\right)=(1+\lambda) \alpha^{F}\left(\sigma^{2}\right)$, it is easy to verify that $x=y$, which provides a contradiction with the starting hypothesis that $\mathcal{P} \subset \Sigma$. Using the same logic one also shows that, as long as the optimal mechanisms features pooling in a subset of $\Sigma$, this subset cannot be the union of multiple disjoint intervals (all strictly inside $\Sigma$ ).

Hence, if $\mathcal{M}_{E}^{*}$ features some pooling, there are only three possible cases to be considered:

(1) $\mathcal{P}=[1-\Delta, x]$ with $1-\Delta<x \leq 1+\Delta$;

(2) $\mathcal{P}=[x, 1+\Delta]$ with $1-\Delta \leq x<1+\Delta$;

(3) $\mathcal{P}=\left[x_{1}, y_{1}\right] \cup\left[x_{2}, y_{2}\right]$ with $1-\Delta=x_{1}<y_{1}<x_{2}<y_{2}=1+\Delta$.

Consider first case (1). The investor's maximization problem is

$$
\max _{\mathcal{P}, \alpha} \mathcal{W}(\mathcal{P}, \alpha) \equiv \max _{x, \alpha}\left\{\int_{1-\Delta}^{x} \alpha\left[\pi-\frac{\alpha \sigma^{2}}{2}\right] d \sigma^{2}+\int_{x}^{1+\Delta} \alpha_{E}^{A}\left(\sigma^{2}\right)\left[\pi-\frac{\alpha_{E}^{A}\left(\sigma^{2}\right) \sigma^{2}}{2}\right] d \sigma^{2}\right\}
$$

The first-order condition with respect to $\alpha$ is

$$
\alpha=\frac{\pi}{\mathbb{E}\left[\sigma^{2} \mid \sigma^{2} \leq x\right]} \equiv \frac{2 \pi}{1-\Delta+x} .
$$

Continuity of the mechanism then implies

$$
\alpha_{E}^{A}(x)=\frac{\pi}{\mathbb{E}\left[\sigma^{2} \mid \sigma^{2} \leq x\right]}
$$

which yields

$$
x_{E}^{*}=\frac{1+\lambda}{1-\lambda}(1-\Delta)
$$

and thus

$$
\alpha_{E}^{*}\left(\sigma^{2}\right)= \begin{cases}\frac{\pi(1-\lambda)}{1-\Delta} & \text { if } \quad \sigma^{2} \leq x_{E}^{*} \\ \frac{\pi(1+\lambda)}{\sigma^{2}} & \text { if } \quad \sigma^{2}>x_{E}^{*}\end{cases}
$$


Finally, $1-\Delta<x_{E}^{*} \leq 1+\Delta$ implies

$$
1-\Delta<\frac{1+\lambda}{1-\lambda}(1-\Delta) \leq 1+\Delta
$$

which requires $\lambda \leq \Delta$. Notice that $x<1+\Delta$ when $\lambda<\Delta$, so that $\mathcal{P}=[1-\Delta, x] \subset \Sigma$. By contrast, $\mathcal{P}=\Sigma$ when $\lambda \geq \Delta$.

Next, we show that an optimal contract cannot satisfies the properties stated in case (2). The proof of this claim is by contradiction. Under case (2), the investor's maximization problem is

$$
\max _{\mathcal{P}, \alpha} \mathcal{W}(\mathcal{P}, \alpha) \equiv \max _{x, \alpha}\left\{\int_{x}^{1+\Delta} \alpha\left[\pi-\frac{\alpha \sigma^{2}}{2}\right] d \sigma^{2}+\int_{1-\Delta}^{x} \alpha_{E}^{A}\left(\sigma^{2}\right)\left[\pi-\frac{\alpha_{E}^{A}\left(\sigma^{2}\right) \sigma^{2}}{2}\right] d \sigma^{2}\right\}
$$

The first-order condition with respect to $\alpha$ is

$$
\alpha=\frac{\pi}{\mathbb{E}\left[\sigma^{2} \mid \sigma^{2} \geq x\right]} \equiv \frac{2 \pi}{x+1+\Delta} .
$$

Continuity of the mechanism then implies

$$
\alpha_{E}^{A}(x)=\frac{\pi}{\mathbb{E}\left[\sigma^{2} \mid \sigma^{2} \geq x\right]}
$$

yielding

$$
x=\frac{1+\lambda}{1-\lambda}(1+\Delta),
$$

Notice that $\lambda>0$ implies $x>1+\Delta$, which contradicts the starting hypothesis $\mathcal{P} \subset \Sigma$. Therefore, an optimal asset allocation cannot satisfy the properties stated in case (2).

Finally, consider case (3). In this scenario the investor pools in two disjoint intervals, say $\mathcal{P}_{1}=[1-$ $\left.\Delta, x_{1}\right]$ and $\mathcal{P}_{2}=\left[x_{2}, 1+\Delta\right]$, with $1-\Delta<x_{1}<x_{2}=1+\Delta$. Let $\mathcal{P}=\left(\mathcal{P}_{1}, \mathcal{P}_{2}\right), \alpha^{\mathcal{P}}=\left(\alpha^{\mathcal{P}_{1}}, \alpha^{\mathcal{P}_{2}}\right)$ and $\mathbf{x}=\left(x_{1}, x_{2}\right)$. The investor's maximization problem is

$$
\begin{aligned}
\max _{\mathcal{P}, \alpha^{\mathcal{P}}} \mathcal{W}\left(\mathcal{P}, \alpha^{\mathcal{P}}\right) \equiv \max _{\mathbf{x}, \alpha^{\mathcal{P}}}\left\{\int_{1-\Delta}^{x_{1}}\right. & \alpha^{\mathcal{P}_{1}}\left[\pi-\frac{\alpha^{\mathcal{P}_{1}} \sigma^{2}}{2}\right] d \sigma^{2}+ \\
& \left.+\int_{x_{1}}^{x_{2}} \alpha_{E}^{A}\left(\sigma^{2}\right)\left[\pi-\frac{\alpha_{E}^{A}\left(\sigma^{2}\right) \sigma^{2}}{2}\right] d \sigma^{2}+\int_{x_{2}}^{1+\Delta} \alpha^{\mathcal{P}_{2}}\left[\pi-\frac{\alpha^{\mathcal{P}_{2}} \sigma^{2}}{2}\right] d \sigma^{2}\right\},
\end{aligned}
$$

where $\alpha^{\mathcal{P}_{1}}$ and $\alpha^{\mathcal{P}_{2}}$ are the shares of wealth invested into the risky asset within the pooling regions $\mathcal{P}_{1}$ and $\mathcal{P}_{2}$, respectively. Optimality then requires

$$
\alpha^{\mathcal{P}_{k}}=\frac{\pi}{\mathbb{E}\left[\sigma^{2} \mid \sigma^{2} \in \mathcal{P}_{k}\right]} \quad k=1,2
$$

Continuity of the mechanism then implies

$$
\frac{\pi}{\mathbb{E}\left[\sigma^{2} \mid \sigma^{2} \leq x_{1}\right]}=\alpha_{E}^{A}\left(x_{1}\right),
$$




$$
\frac{\pi}{\mathbb{E}\left[\sigma^{2} \mid \sigma^{2} \geq x_{2}\right]}=\alpha_{E}^{A}\left(x_{2}\right)
$$

Solving for $x_{1}$ and $x_{2}$

$$
\begin{gathered}
x_{1}=\frac{1+\lambda}{1-\lambda}(1-\Delta), \\
x_{2}=\frac{1+\lambda}{1-\lambda}(1+\Delta)>1+\Delta,
\end{gathered}
$$

which is a contradiction. Therefore, an optimal asset allocation cannot satisfy the properties stated in case (3).

In order to complete the characterization of the optimal mechanism we need to show that the investor never gains from full delegation - i.e., $\mathcal{P}=\varnothing$ cannot be an optimum. Specifically, for any asset allocation rule that satisfies (1) the following holds

$$
\left.\frac{\partial \mathcal{W}(x, \alpha(x))}{\partial x}\right|_{x=1-\Delta}=\frac{\gamma \lambda^{2} \pi^{2}}{2(1-\Delta)}>0
$$

where

$$
\alpha(x) \equiv \frac{2 \pi}{1-\Delta+x} .
$$

But this directly implies $x>1-\Delta$. Finally, notice that $\mathcal{W}(x, \alpha)$ is concave in $\alpha$. In fact, substituting for $\alpha_{E}^{*}(x)$ we have

$$
\left.\frac{\partial^{2} \mathcal{W}(x, \alpha(x))}{\partial x^{2}}\right|_{x=x_{E}^{*}}=-\frac{\gamma}{2} \pi^{2} \frac{(1-\lambda)^{3}}{(1-\Delta)^{2}(1+\lambda)}(2 \lambda+1)<0
$$

which completes the proof.

Proof of Lemma 1. The proof of the lemma is by contradiction.

To begin with, we show that there exists no symmetric equilibrium where both investors offer a mechanism $\mathcal{M}$ that requires pooling in the subset $\mathcal{P} \subset \Sigma$. Let $\mathcal{P} \equiv[x, y]$, with $1-\Delta<x<y<1+\Delta$, be one of the (disjoint) regions in which both investors pool at equilibrium - i.e., the subset of $\Sigma$ in which each investor chooses $\alpha$ for every $\sigma^{2} \in \mathcal{P}$. Hence, outside $\mathcal{P}$ there must exist some intervals of $\Sigma$ in which both investors delegate their portfolio choices to the advisor, thus allocating a fraction $\alpha_{N}^{A}\left(\sigma^{2}\right)$ of their wealth into the risky asset.

Continuity of the mechanism $\mathcal{M}$ then implies $\alpha_{N}^{A}(x)=\alpha_{N}^{A}(y)=\alpha$, where optimality requires

$$
\alpha=\frac{\pi}{\mathbb{E}\left[\sigma^{2} \mid \sigma^{2} \in \mathcal{P}\right]} .
$$

Using (.6) and the expression for $\alpha_{N}^{A}\left(\sigma^{2}\right)$ this system of equations can be rewritten as

$$
\begin{aligned}
& \alpha_{N}^{A}(x)=\frac{2 \pi}{x+y}, \\
& \alpha_{N}^{A}(y)=\frac{2 \pi}{x+y},
\end{aligned}
$$

whose unique solution requires $x=y$, which contradicts $\mathcal{P} \subset \Sigma$.

Next, we prove the remaining part of the Lemma. First, notice that the above argument rules out the possibility of having multiple (disjoint) delegation regions strictly contained in $\Sigma$. Otherwise, we 
should have at least one pooling region strictly contained in $\Sigma$. However, this does not exclude the existence of a single delegation region strictly inside $\Sigma$, surrounded by two pooling regions. In what follows we show that this cannot be possible as well. The proof is again by contradiction. Suppose that such a symmetric equilibrium exists. Accordingly, let $\mathcal{P}_{1} \equiv[1-\Delta, x]$ and $\mathcal{P}_{2} \equiv[y, 1+\Delta]$, with $1-\Delta<x<y<1+\Delta$, be the two regions where both investors pool - i.e., the subsets of $\Sigma$ in which each investor chooses $\alpha^{\mathcal{P}_{1}}$ for every $\sigma^{2} \in \mathcal{P}_{1}$ and $\alpha^{\mathcal{P}_{2}}$ for every $\sigma^{2} \in \mathcal{P}_{2}$. On the contrary, delegation takes place in the interior $[x, y]$.

Continuity of the mechanism $\mathcal{M}$ then implies $\alpha_{N}^{A}(x)=\alpha^{\mathcal{P}_{1}}$ and $\alpha_{N}^{A}(y)=\alpha^{\mathcal{P}_{2}}$ - where, optimality of the mechanism requires

$$
\begin{aligned}
\alpha^{\mathcal{P}_{1}} & =\frac{\pi}{\mathbb{E}\left[\sigma^{2} \mid \sigma^{2} \in \mathcal{P}_{1}\right]}, \\
\alpha^{\mathcal{P}_{2}} & =\frac{\pi}{\mathbb{E}\left[\sigma^{2} \mid \sigma^{2} \in \mathcal{P}_{2}\right]} .
\end{aligned}
$$

Hence

$$
x=\frac{1+\lambda}{1-\lambda+2 \theta}(1-\Delta), \quad y=\frac{1+\lambda}{1-\lambda+2 \theta}(1+\Delta),
$$

which clearly contradict the starting hypothesis that $1-\Delta<x$ and $y<1+\Delta$.

Proof of Proposition 2. The proof of the proposition is structured as follows. First, we characterize the properties that a symmetric (candidate) equilibrium where both investors pool for low values of $\sigma^{2}$ and delegate otherwise need to satisfy. Second, we show that, in the region of parameters under consideration, this outcome is immune from unilateral deviations within the class of continuous mechanisms. Third, we show that there exists a non-empty region of parameters where there exists an equilibrium with full pooling. Finally, we argue that within the regions of parameters under consideration there are no other symmetric equilibria.

Suppose that both investors offer a mechanism $\mathcal{M}_{N}^{*}=\left\{\alpha_{N}^{*}\left(\sigma^{2}\right)\right\}_{\sigma^{2} \in \Sigma}$ that entails a pooling allocation in the subset $\mathcal{P}_{N}^{*} \equiv\left[1-\Delta, x_{N}^{*}\right] \subseteq \Sigma$, with $1-\Delta<x_{N}^{*}<1+\Delta$, and a separating one for $\sigma^{2}>x_{N}^{*}$ - i.e.,

$$
\alpha_{N}^{*}\left(\sigma^{2}\right)=\left\{\begin{array}{lll}
\alpha_{N}^{*} & \text { if } & \sigma^{2} \leq x_{N}^{*} \\
\alpha_{N}^{A}\left(\sigma^{2}\right) & \text { if } & \sigma^{2}>x_{N}^{*}
\end{array} .\right.
$$

The first-order condition identifying $\alpha_{N}^{*}$ is

$$
\alpha_{N}^{*}=\frac{\pi}{\mathbb{E}\left[\sigma^{2} \mid \sigma^{2} \in \mathcal{P}_{N}^{*}\right]}
$$

While continuity of $\mathcal{M}_{N}^{*}$ requires $\alpha_{N}^{*}=\alpha_{N}^{A}\left(x_{N}^{*}\right)$. Substituting (.7) and the expression for $\alpha_{N}^{A}\left(\sigma^{2}\right)$ into this equation, $x_{N}^{*}$ solves

$$
\frac{x_{N}^{*}}{\mathbb{E}\left[\sigma^{2} \mid \sigma^{2} \leq x_{N}^{*}\right]}=\frac{1+\lambda}{1+\theta},
$$

yielding

$$
x_{N}^{*}=\frac{1+\lambda}{1-\lambda+2 \theta}(1-\Delta)
$$

and thus

$$
\alpha_{N}^{*}=\pi \frac{1-\lambda+2 \theta}{(1+\theta)(1-\Delta)}
$$

Notice that $x_{N}^{*} \in(1-\Delta, 1+\Delta)$ in the region of parameters under consideration. Following the approach of Proposition 1 concavity of the investors' expected utility at $\left(x_{N}^{*}, \alpha_{N}^{*}\left(\sigma^{2}\right)\right)$ can be easily checked. Hence, $\theta>(\lambda-\Delta) /(1+\Delta)$ and $\lambda<\Delta$ are necessary conditions for such a symmetric equilibrium to 
exist. In the following we show that they are also sufficient.

Next, we show that, within the class of continuous mechanisms and incentive compatible mechanisms, there are no profitable deviations from the symmetric outcome characterized above. The proof is developed in the following steps, where it is assumed (without loss of generality) that investor 1 sticks to the equilibrium behavior.

Step 1. Consider first the class of deviations where investor 2 offers a mechanism $\mathcal{M}_{2}$ such that $\alpha_{2}($. is constant in a neighborhood of $\sigma^{2}=1-\Delta$ - i.e., there exists a non-empty neighborhood of $1-\Delta$, say $\mathcal{B}(1-\Delta)$, such that $\mathcal{B}(1-\Delta) \subseteq \Sigma$ and $\dot{\alpha}_{2}\left(\sigma^{2}\right)=0$ for every $\sigma^{2} \in \mathcal{B}(1-\Delta)$.

1.A. Suppose that investor 2 deviates by offering a mechanism $\mathcal{M}_{2}$ that entails pooling only in region $\mathcal{P}_{2} \equiv[1-\Delta, x]$, with $1-\Delta<x<1+\Delta$ and $x \neq x_{N}^{*}$.

Showing that this deviation is unprofitable in the region of parameters under consideration is straightforward. Indeed, continuity of the optimal mechanism implies $x=x_{N}^{*}$. This is immediate for $x>x_{N}^{*}$. By contrast, for $x<x_{N}^{*}$ continuity of $\mathcal{M}_{2}$ requires

$$
\frac{\pi}{\mathbb{E}\left[\sigma^{2} \mid \sigma^{2} \leq x\right]}=\alpha_{E}^{A}(x)-\theta \alpha_{N}^{*},
$$

which has only one solution in $\Sigma$ equal to $x_{N}^{*}$. A contradiction.

1.B. Suppose that investor 2 deviates by offering a mechanism $\mathcal{M}_{2}$ that entails pooling in regions $\mathcal{P}_{2} \equiv[1-\Delta, x]$ and $\mathcal{P}_{2}^{\prime}=[y, 1+\Delta]$, with $y>x \geq x_{N}^{*}$.

Continuity of $\mathcal{M}_{2}$ requires

$$
\begin{gathered}
\frac{\pi}{\mathbb{E}\left[\sigma^{2} \mid \sigma^{2} \leq x\right]}=\alpha_{N}^{A}(x) \quad \Leftrightarrow \quad x=x_{N}^{*}, \\
\frac{\pi}{\mathbb{E}\left[\sigma^{2} \mid \sigma^{2} \geq y\right]}=\alpha_{N}^{A}(y) \quad \Leftrightarrow \quad y=\frac{1+\lambda}{1-\lambda+2 \theta}(1+\Delta) .
\end{gathered}
$$

But, in the region of parameters under consideration, it is easy to verify that $y>1+\Delta$. A contradiction.

1.C. Suppose that investor 2 deviates by offering a mechanism $\mathcal{M}_{2}$ that entails pooling in regions $\mathcal{P}_{2} \equiv[1-\Delta, x]$ and $\mathcal{P}_{2}^{\prime}=[y, 1+\Delta]$, with $1+\Delta>y>x_{N}^{*}>x>1-\Delta$.

In this case the same contradiction obtained in step 1.B obtains.

1.D. Suppose that investor 2 deviates by offering a mechanism $\mathcal{M}_{2}$ that entails pooling in regions $\mathcal{P}_{2} \equiv[1-\Delta, x]$ and $\mathcal{P}_{2}^{\prime}=[y, 1+\Delta]$, with $1+\Delta>x_{N}^{*} \geq y>x>1-\Delta$.

Continuity of $\mathcal{M}_{2}$ requires

$$
\begin{aligned}
& \frac{\pi}{\mathbb{E}\left[\sigma^{2} \mid \sigma^{2} \leq x\right]}=\alpha_{E}^{A}(x)-\theta \alpha_{N}^{*}, \\
& \frac{\pi}{\mathbb{E}\left[\sigma^{2} \mid \sigma^{2} \geq y\right]}=\alpha_{E}^{A}(y)-\theta \alpha_{N}^{*} .
\end{aligned}
$$

Hence

$$
\begin{aligned}
& \frac{2}{1-\Delta+x}=\frac{1+\lambda}{x}-\theta \frac{1-\lambda+2 \theta}{(1+\theta)(1-\Delta)}, \\
& \frac{2}{1+\Delta+y}=\frac{1+\lambda}{y}-\theta \frac{1-\lambda+2 \theta}{(1+\theta)(1-\Delta)} .
\end{aligned}
$$

Notice that, in the region of parameters under consideration, (.8) has two solutions: one negative and one equal to $x_{N}^{*}$. A contradiction with the initial assumption that $x_{N}^{*} \geq y>x$. 
1.E. Suppose that investor 2 deviates by offering a mechanism $\mathcal{M}_{2}$ that entails full pooling. In order to show that this cannot be a best reply, consider the case where investor 2 deviates by using a strategy like the ones considered in 1.A. Clearly, full pooling is a degenerated form of this class of strategy, where $x=1+\Delta$. But, this corner solution can be optimal if and only if the derivative of the unconstrained maximization problem of investor 2 with respect to $x$ is non negative at $x=1+\Delta$. Recall that if $x=1+\Delta$, then optimality requires investor 2 to invest $\pi$ into the risky asset for every $\sigma^{2}$. Hence, the derivative of investor 2's expected utility with respect to $x$ evaluated at $x=1+\Delta$ is

$$
\begin{aligned}
\gamma\left[\pi-\alpha_{N}^{A}(1+\Delta)\right] \times\left[\pi-\frac{1}{2}\left[\alpha_{N}^{A}(1+\Delta)+\pi\right](1+\Delta)\right] & = \\
& -\gamma \pi^{2} \frac{\Delta-\lambda+\theta+\Delta \theta}{(1+\theta)(1+\Delta)} \times \frac{\Delta(1+\theta)+\lambda-\theta}{2(1+\theta)},
\end{aligned}
$$

which is strictly negative in the region of parameters under consideration. A contradiction.

1.F. Finally, using the same arguments developed in the proof of cases $\mathbf{1 . B}, \mathbf{1 . C}$, and $\mathbf{1 . D}$ it is easy to verify that deviations starting with a pooling allocation at $1-\Delta$ and involving at least two disjoint separation regions are not profitable.

Step 2. Consider now the class of deviations where investor 2 offers a mechanism $\mathcal{M}_{2}$ such that $\alpha_{2}($. is (strictly) decreasing in a neighborhood of $\sigma^{2}=1-\Delta$ - i.e., there exists a non-empty neighborhood of $1-\Delta$, say $\mathcal{B}(1-\Delta)$, such that $\mathcal{B}(1-\Delta) \subseteq \Sigma$ and $\dot{\alpha}_{2}\left(\sigma^{2}\right)<0$ for every $\sigma^{2} \in \mathcal{B}(1-\Delta)$.

2.A. Suppose that investor 2 deviates by offering a mechanism $\mathcal{M}_{2}$ that entails pooling only in region $\mathcal{P}_{2} \equiv[x, 1+\Delta]$, with $1-\Delta<x_{N}^{*} \leq x<1+\Delta$.

Continuity of $\mathcal{M}_{2}$ requires

$$
\alpha_{N}^{A}(x)=\frac{\pi}{\mathbb{E}\left[\sigma^{2} \mid \sigma^{2} \geq x\right]} \quad \Leftrightarrow \quad x=\frac{1+\lambda}{1-\lambda+2 \theta}(1+\Delta) .
$$

By construction $x<1+\Delta$, which would imply $\lambda<\theta$. A contradiction.

2.B. Suppose that investor 2 deviates by offering a mechanism $\mathcal{M}_{2}$ that entails pooling only in region $\mathcal{P}_{2} \equiv[x, 1+\Delta]$, with $1-\Delta<x<x_{N}^{*}<1+\Delta$.

Continuity of $\mathcal{M}_{2}$ requires

$$
\frac{\pi}{\mathbb{E}\left[\sigma^{2} \mid \sigma^{2} \geq x\right]}=\alpha_{E}^{A}(x)-\theta \alpha_{N}^{*},
$$

that is

$$
\frac{2 \pi}{x+1+\Delta}=(1+\lambda) \frac{\pi}{x}-\theta \alpha_{N}^{*}
$$

Let

$$
\Phi(z) \equiv(1+\lambda) \frac{\pi}{z}-\frac{2 \pi}{z+1+\Delta} .
$$

Condition (.10) then rewrites as $\Phi(x)=\theta \alpha_{N}^{*}$. Notice that

$$
\Phi(1-\Delta)=\pi \frac{\lambda+\Delta}{1-\Delta}>\Phi(1+\Delta)=\frac{\pi \lambda}{1+\Delta}>0
$$

and

$$
\Phi^{\prime}(z)=-\pi \frac{(1+\Delta)^{2}(1+\lambda)-z^{2}(1-\lambda)+2 z(1+\Delta)(1+\lambda)}{z^{2}(\Delta+1+z)^{2}}
$$


where it can be verified that

$$
(1+\Delta)^{2}(1+\lambda)-z^{2}(1-\lambda)+2 z(1+\Delta)(1+\lambda)>0 \quad \forall z \in \Sigma .
$$

Hence, $\Phi^{\prime}(z)<0$ in $\Sigma$. Taken together, these conditions imply that $\Phi(x)>0$ in $\Sigma$.

Next, note that for $\theta<0,(.10)$ has no solution, which yields a contradiction.

2.C. Suppose that investor 2 deviates by offering a mechanism $\mathcal{M}_{2}$ that entails pooling only in region $\mathcal{P}_{2} \equiv[x, y]$, with $1-\Delta<x<y<1+\Delta$.

First, it is straightforward to show that continuity of the mechanism (together with optimality) rules out deviations such that $y>x>x_{N}^{*}$ and $x_{N}^{*}>y>x$.

Next, consider a deviation such that $x<x_{N}^{*}<y$. Continuity of $\mathcal{M}_{2}$ imply

$$
\begin{gathered}
\frac{\pi}{\mathbb{E}\left[\sigma^{2} \mid \sigma^{2} \in \mathcal{P}_{2}\right]}=\alpha_{E}^{A}(x)-\theta \alpha_{N}^{*}, \\
\frac{\pi}{\mathbb{E}\left[\sigma^{2} \mid \sigma^{2} \in \mathcal{P}_{2}\right]}=\alpha_{N}^{A}(y) .
\end{gathered}
$$

Hence,

$$
\begin{gathered}
\frac{2 \pi}{x+y}=(1+\lambda) \frac{\pi}{x}-\theta \alpha_{N}^{*}, \\
\frac{2 y}{x+y}=\frac{1+\lambda}{1+\theta} .
\end{gathered}
$$

The solution of this system of equations is

$$
\begin{gathered}
x=\frac{2 \lambda-\theta(1-\lambda)}{(1-\lambda+2 \theta) \theta}(1-\Delta), \\
y=\frac{(2 \lambda-\theta(1-\lambda))(1+\lambda)}{(1-\lambda+2 \theta)^{2} \theta}(1-\Delta) .
\end{gathered}
$$

Notice that

$$
x-(1-\Delta)=\frac{2(\lambda-\theta)(1+\theta)}{(1-\lambda+2 \theta) \theta}(1-\Delta)<0 \quad \Leftrightarrow \quad \theta<0 .
$$

Hence, for $\theta<0$, this yields a contradiction in the region of parameters under consideration.

2.D. Suppose that investor 2 deviates by offering a mechanism $\mathcal{M}_{2}$ that entails full delegation.

Notice that, given the mechanism offered by investor 1, the advisor's ideal point over investor 2's asset allocation choice is

$$
\alpha_{2}^{A}\left(\sigma^{2}\right)=\left\{\begin{array}{llc}
\alpha_{N}^{A}\left(\sigma^{2}\right) & \text { if } & \sigma^{2} \geq x_{N}^{*} \\
\alpha_{E}^{A}\left(\sigma^{2}\right)-\theta \alpha_{N}^{*} & \text { if } \quad \sigma^{2}<x_{N}^{*}
\end{array} .\right.
$$

Hence, $\theta \leq 0$ implies $\alpha_{2}^{A}\left(\sigma^{2}\right)>\alpha^{F}\left(\sigma^{2}\right)$. Then, using the same logic of the proof of Proposition 1, it follows that for investor 2 it is optimal to pool in a non-empty subset of $\Sigma$. A contradiction.

2.E. Finally, using the same arguments developed in the proof of cases $\mathbf{2 . A , 2 . B}$, and 2.C, it can be verified that deviations starting with a separating at $\sigma^{2}=1-\Delta$ and involving at least two disjoint pooling regions are not profitable. 
We now turn to characterize the region of parameters where there exists pooling equilibrium. Recall that in an equilibrium with full pooling both investors choose $\alpha_{N}^{*}\left(\sigma^{2}\right)=\pi$ regardless of the advisor's reports. We must then show that there exists a region of parameters where there are no profitable deviations from this outcome. As before, assume (without loss of generality) that investor 1 pools i.e., $\alpha_{1}=\pi \forall m \in \Sigma$. Consider a deviation by investor 2 such that

$$
\alpha_{2}\left(\sigma^{2}\right)=\left\{\begin{array}{lll}
\bar{\alpha}_{2} & \text { if } & \sigma^{2}<\underline{x} \\
\alpha_{2}^{A}\left(\sigma^{2}\right) & \text { if } & \sigma^{2} \in[\underline{x}, \bar{x}] \\
\underline{\alpha}_{2} & \text { if } & \sigma^{2}>\bar{x}
\end{array},\right.
$$

where $1+\Delta \geq \bar{x} \geq \underline{x} \geq 1-\Delta$. Notice that incentive compatibility implies $\bar{\alpha}_{2}>\underline{\alpha}_{2}$ and

$$
\alpha_{2}^{A}\left(\sigma^{2}\right)=\alpha_{E}^{A}\left(\sigma^{2}\right)-\theta \pi
$$

Notice that whenever $\underline{x} \geq 1+\Delta$, investor 2 's best reaction entails full pooling. If this is not the case, one must have that the solution of investor 2's maximization problem requires $1+\Delta \geq \bar{x}>\underline{x} \geq 1-\Delta$. In what follows we show that in the region of parameters where $\theta \leq(\lambda-\Delta) /(1+\Delta)$ this is not possible.

The derivative of investor 2's expected utility with respect to $\underline{x}$ is

$$
\gamma\left[\bar{\alpha}_{2}-\alpha_{2}^{A}(\underline{x})\right] \times\left[\pi-\frac{1}{2}\left[\alpha_{2}^{A}(\underline{x})+\bar{\alpha}_{2}\right] \underline{x}\right],
$$

where

$$
\bar{\alpha}_{2}=\frac{\pi}{\mathbb{E}\left[\sigma^{2} \mid \sigma^{2} \leq \underline{x}\right]} .
$$

Evaluating (.12) at $\underline{x}=1+\Delta$, so that $\bar{\alpha}_{2}=\pi$, this derivative can be rewritten as

$$
\gamma \pi^{2}\left[\frac{\lambda-\Delta(1+\theta)-\theta}{1+\Delta}\right] \times\left[\frac{\lambda+\Delta(1-\theta)-\theta}{2}\right]
$$

By the same token, the derivative of investor 2's expected utility with respect to $\bar{x}$ is

$$
\gamma\left[\alpha_{2}^{A}(\bar{x})-\underline{\alpha}_{2}\right] \times\left[\pi-\frac{1}{2}\left[\alpha_{2}^{A}(\bar{x})+\underline{\alpha}_{2}\right] \bar{x}\right]
$$

where

$$
\underline{\alpha}_{2}=\frac{\pi}{\mathbb{E}\left[\sigma^{2} \mid \sigma^{2} \geq \bar{x}\right]} .
$$

Evaluating (.14) at $\bar{x}=1-\Delta$ so that $\underline{\alpha}_{2}=\pi$, this derivative can be rewritten as

$$
-\gamma \pi^{2}\left[\frac{\lambda-\theta(1+\Delta)+\Delta}{1-\Delta}\right] \times\left[\frac{\lambda-\Delta(1-\theta)-\theta}{2}\right]
$$

In the region of parameters under consideration, the expression in equation (.13) is positive and that in equation (.15) is negative. Hence, by concavity of investor 2's objective function (which can be immediately checked) it follows that investor 2's best reply to full pooling by investor 1 is full pooling, which shows that full pooling is an equilibrium in this region.

Moreover, notice that a strategy for investor 2 that requires a pooling allocation only when $\sigma^{2} \in$ int $\Sigma$ is not feasible: this would indeed contradict continuity of the optimal mechanisms. Hence, in the region 
of parameters under consideration there is a unique symmetric equilibrium where both investors pool. This result, together with the first part of the proof also implies that in each of the two regions of parameters identified by the statement of the proposition there is a unique symmetric equilibrium.

Proof of Proposition 3. The proof of the proposition is structured as follows. As before, we first characterize the properties that a symmetric (candidate) equilibrium where both investors pool for high values of $\sigma^{2}$ and delegate otherwise need to satisfy. Second, we show that, in the region of parameters under consideration, this outcome is immune from unilateral deviations within the class of continuous mechanisms. Third, we show that there exists a non-empty region of parameters where there exists an equilibrium with full pooling. Finally, we argue that within the regions of parameters under consideration there are no other symmetric equilibria.

To begin with, notice that when $\lambda=\theta$ there is a unique symmetric equilibrium with full delegation. Second, it can be checked that the proof of Proposition 2 can be extended to the case where $\lambda>\theta>0$. Hence, for brevity we will focus on the novel type of equilibrium that emerges when $\theta>\lambda$.

Suppose that both investors offer a mechanism $\mathcal{M}_{N}^{*} \equiv\left\{\alpha_{N}^{*}\left(\sigma^{2}\right)\right\}_{\sigma^{2} \in \Sigma}$ that entails a pooling allocation in the subset $\mathcal{P}_{N}^{*} \equiv\left[x_{N}^{*}, 1+\Delta\right] \subseteq \Sigma$, with $1-\Delta<x_{N}^{*}<1+\Delta$, and a separating one for $\sigma^{2}<x_{N}^{*}$ - i.e.,

$$
\alpha_{N}^{*}\left(\sigma^{2}\right)=\left\{\begin{array}{lll}
\alpha_{N}^{A}\left(\sigma^{2}\right) & \text { if } & \sigma^{2} \leq x_{N}^{*} \\
\alpha_{N}^{*} & \text { if } & \sigma^{2}>x_{N}^{*}
\end{array} .\right.
$$

From the optimality conditions of the investors' maximization problem, it follows that

$$
\alpha_{N}^{*}=\frac{\pi}{\mathbb{E}\left[\sigma^{2} \mid \sigma^{2} \in \mathcal{P}\right]} \equiv \frac{2 \pi}{x_{N}^{*}+1+\Delta},
$$

while continuity of the mechanism implies that $\alpha_{N}^{*}=\alpha_{N}^{A}\left(x_{N}^{*}\right)$. Substituting (.16) and the expression for $\alpha_{N}^{A}\left(\sigma^{2}\right)$, this equation rewrites as

$$
\frac{2 \pi}{x_{N}^{*}+1+\Delta}-\alpha_{N}^{A}\left(x^{*}\right)=0
$$

whose unique solution yields

$$
x_{N}^{*}=\frac{1+\lambda}{1-\lambda+2 \theta}(1+\Delta)
$$

Therefore, the pooling allocation is

$$
\alpha_{N}^{*}=\pi \frac{1-\lambda+2 \theta}{(1+\theta)(1+\Delta)}
$$

Since $\lambda<1$, it follows that $x_{N}^{*} \in(1-\Delta, 1+\Delta)$ in the region of parameters where $\lambda<\theta<$ $(\lambda+\Delta) /(1-\Delta)$. As before, it can be shown that concavity of the investors' expected utility holds at $\left(x_{N}^{*}, \alpha_{N}^{*}\right)$ within the region of parameters under consideration. Hence, $\lambda<\theta<(\lambda+\Delta) /(1-\Delta)$ is a necessary condition for such a symmetric equilibrium to exist. In the following we show that they are also sufficient.

Next, we show that, within the class of continuous mechanisms and incentive compatible mechanisms, there are no profitable deviations from the symmetric outcome characterized above. The proof is developed in the following steps, where it is assumed (without loss of generality) that investor 1 sticks to the equilibrium behavior.

Step 1. Consider first the class of deviations where investor 2 offers a mechanism $\mathcal{M}_{2}$ such that $\alpha_{2}($. 
is constant in a neighborhood of $\sigma^{2}=1+\Delta$.

1.A. Suppose that investor 2 deviates by offering a mechanism $\mathcal{M}_{2}$ that entails pooling only in region $\mathcal{P}_{2} \equiv[x, 1+\Delta]$, with $1-\Delta<x_{N}^{*}<1+\Delta$ and $x \neq x_{N}^{*}$.

Showing that this deviation is unprofitable in the region of parameters under consideration is straightforward. Indeed, continuity of the optimal mechanism implies $x=x_{N}^{*}$. This is immediate for $x<x_{N}^{*}$. By contrast, for $x>x_{N}^{*}$ continuity of of $\mathcal{M}_{2}$ requires

$$
\frac{\pi}{\mathbb{E}\left[\sigma^{2} \mid \sigma^{2} \geq x\right]}=\alpha_{E}^{A}(x)-\theta \alpha_{N}^{*},
$$

which has only one solution in $\Sigma$ equal to $x_{N}^{*}$. A contradiction.

1.B. Suppose that investor 2 deviates by offering a mechanism $\mathcal{M}_{2}$ that entails pooling in regions $\mathcal{P}_{2} \equiv[1-\Delta, x]$ and $\mathcal{P}_{2}^{\prime}=[y, 1+\Delta]$, with $x<y \leq x_{N}^{*}$.

By continuity, the optimal mechanism $\mathcal{M}_{2}$ must satisfy

$$
\begin{gathered}
\frac{2 \pi}{1-\Delta+x}=\alpha_{N}^{A}(x) \quad \Leftrightarrow \quad x=\frac{1+\lambda}{1-\lambda+2 \theta}(1-\Delta), \\
\frac{2 \pi}{1+\Delta+y}=\alpha_{N}^{A}(y) \Leftrightarrow y=x_{N}^{*} .
\end{gathered}
$$

But, in the region of parameters under consideration it is easy to verify that $x<1-\Delta$ : a contradiction.

1.C. Suppose that investor 2 deviates by offering a mechanism $\mathcal{M}_{2}$ that entails pooling in regions $\mathcal{P}_{2} \equiv[1-\Delta, x]$ and $\mathcal{P}_{2}^{\prime}=[y, 1+\Delta]$, with $1+\Delta>y>x_{N}^{*}>x>1-\Delta$.

In this case the same contradiction obtained in step 1.B obtains.

1.D. Suppose that investor 2 deviates by offering a mechanism $\mathcal{M}_{2}$ that entails a pool allocation in regions $\mathcal{P}_{2} \equiv[1-\Delta, x]$ and $\mathcal{P}_{2}^{\prime}=[y, 1+\Delta]$, with $1+\Delta>y>x \geq x_{N}^{*}>1-\Delta$.

By continuity, the optimal mechanism $\mathcal{M}_{2}$ must satisfy

$$
\begin{aligned}
& \frac{2 \pi}{\mathbb{E}\left[\sigma^{2} \mid \sigma^{2} \leq x\right]}=\alpha_{E}^{A}(x)-\theta \alpha_{N}^{*}, \\
& \frac{2 \pi}{\mathbb{E}\left[\sigma^{2} \mid \sigma^{2} \geq y\right]}=\alpha_{E}^{A}(y)-\theta \alpha_{N}^{*} .
\end{aligned}
$$

Hence, $x$ and $y$ must solve

$$
\begin{aligned}
& \frac{2}{1-\Delta+x}=\frac{1+\lambda}{x}-\theta \frac{1-\lambda+2 \theta}{(1+\theta)(1+\Delta)}, \\
& \frac{2}{1+\Delta+y}=\frac{1+\lambda}{y}-\theta \frac{1-\lambda+2 \theta}{(1+\theta)(1+\Delta)} .
\end{aligned}
$$

Notice that, in the region of parameters under consideration, the latter equation has a unique positive solution $y=x_{N}^{*}$. A contradiction with the initial assumption that $y>x \geq x_{N}^{*}$.

1.E. Suppose that investor 2 deviates by offering a mechanism $\mathcal{M}_{2}$ that entails full pooling. In order to show that this is not optimal, consider the case where investor 2 deviates by using a strategy like the ones considered in 1.A. Clearly, full pooling is a degenerated form of this class of strategy, where $x=1-\Delta$. But, this corner solution can be optimal if and only if the derivative of the unconstrained maximization problem of investor 2 with respect to $x$ is non positive at $x=1-\Delta$. Recall that if $x=1-\Delta$, then optimality requires $\alpha_{2}^{*}=\pi$ for every $\sigma^{2}$. The derivative of investor 2 's expected utility 
with respect to $x$ evaluated at $x=1-\Delta$ is

$$
\begin{aligned}
\gamma\left[\alpha_{N}^{A}(1-\Delta)-\pi\right] \times\left[\pi-\frac{1}{2}\left[\alpha_{N}^{A}(1-\Delta)+\pi\right](1-\Delta)\right] & = \\
& \gamma \pi^{2} \frac{(\Delta+\lambda-\theta+\Delta \theta)}{(1+\theta)(1-\Delta)} \frac{(\Delta(1+\theta)-\lambda+\theta)}{2(1+\theta)}>0,
\end{aligned}
$$

which is strictly in the region of parameters under consideration: a contradiction.

1.F. Using the same arguments developed in the proof of cases 1.B, 1.C and 1.D, it is easy to verify that deviations starting with a pooling at $1+\Delta$ and involving at least two disjoint separation regions are not profitable.

Step 2. Consider now the class of deviations where investor 2 offers a mechanism $\mathcal{M}_{2}=\left\{\alpha_{2}\left(\sigma^{2}\right)\right\}_{\sigma^{2} \in \Sigma}$ such that $\alpha_{2}($.$) is (strictly) decreasing in a neighborhood of \sigma^{2}=1+\Delta-$ i.e., there exists a nonempty neighborhood of $1+\Delta$, say $\mathcal{B}(1+\Delta)$, such that $\mathcal{B}(1+\Delta) \subseteq \Sigma$ and $\dot{\alpha}_{2}\left(\sigma^{2}\right)<0$ for every $\sigma^{2} \in \mathcal{B}(1+\Delta)$.

2.A. Suppose that investor 2 deviates by offering a mechanism $\mathcal{M}_{2}$ that entails pooling only in region $\mathcal{P}_{2} \equiv[1-\Delta, x]$, with $1-\Delta<x \leq x_{N}^{*}<1+\Delta$.

Continuity of the mechanism, together with optimality, imply

$$
\alpha_{N}^{A}(x)=\frac{2 \pi}{1-\Delta+x} \quad \Leftrightarrow \quad x=\frac{1+\lambda}{1-\lambda+2 \theta}(1-\Delta) .
$$

By construction $x>1-\Delta$, which requires $\lambda>\theta$. But, this condition is not met in the region of parameters under consideration.

2.B. Suppose that investor 2 deviates by offering a mechanism $\mathcal{M}_{2}$ that entails pooling only in region $\mathcal{P}_{2} \equiv[1-\Delta, x]$, with $1-\Delta<x_{N}^{*}<x<1+\Delta$.

Continuity of the mechanism, together with optimality, imply

$$
\alpha_{E}^{A}(x)-\theta \alpha_{N}^{*}=\frac{2 \pi}{1-\Delta+x}
$$

Define

$$
\Phi(z) \equiv \alpha_{E}^{A}(z)-\frac{2 \pi}{1-\Delta+z} .
$$

Condition (.17) rewrites as $\Phi(x)=\theta \alpha_{N}^{*}$. Notice that

$$
\begin{gathered}
\Phi(1-\Delta)=\frac{\pi \lambda}{1-\Delta}>0, \quad \Phi(1+\Delta)=\pi \frac{\lambda-\Delta}{1+\Delta} \\
\Phi^{\prime}(z)=-\pi \frac{(1-\Delta)^{2}(1+\lambda)-z^{2}(1-\lambda)+2 z(1-\Delta)(1+\lambda)}{z^{2}(z+1-\Delta)^{2}}
\end{gathered}
$$

and

$$
\Phi\left(x_{N}^{*}\right)-\theta \alpha_{N}^{*}=-\frac{(1-\lambda+2 \theta)^{2} \Delta \pi}{(1+\theta(1-\Delta)+\Delta \lambda)(1+\theta)(1+\Delta)}<0 .
$$

Moreover, $\Phi(1+\Delta)>0$ and $\Phi^{\prime}(z)<0$ for every $z \in \Sigma$ if $\lambda>\Delta$. Hence, $\Phi\left(x_{N}^{*}\right)<\theta \alpha_{N}^{*}$ directly implies that $x_{N}^{*}>x$ for $\lambda>\Delta$ : a contradiction. Next, suppose that $\lambda \leq \Delta$. In this region of parameters it is easy to verify that $\Phi^{\prime}(z)=0$ has a unique solution in $\Sigma$ (say $z^{*}$ ) with $\Phi^{\prime}(z)>0$ if and only if $z>z^{*}$ 
and $\Phi\left(z^{*}\right)<0$. Hence, $\Phi(1+\Delta) \leq 0$ together with $\Phi\left(x_{N}^{*}\right)<\theta \alpha_{N}^{*}$, directly imply that $x_{N}^{*}>x a$ fortiori when $\lambda \leq \Delta$ : again a contradiction.

2.C. Suppose that investor 2 deviates by offering a mechanism $\mathcal{M}_{2}$ that entails pooling only in region $\mathcal{P}_{2} \equiv[x, y]$, with $1-\Delta<x<y<1+\Delta$.

First, it is straightforward to show that continuity of the mechanism (together with optimality) rules out deviations such that $y>x>x_{N}^{*}$ and $x_{N}^{*}>y>x$.

Next, consider a deviation such that $x<x_{N}^{*}<y$. Continuity of $\mathcal{M}_{2}$, together with optimality, imply

$$
\begin{gathered}
\alpha_{N}^{A}(x)=\frac{2 \pi}{x+y}, \\
\alpha_{E}^{A}(y)-\theta \alpha_{N}^{*}=\frac{2 \pi}{x+y} .
\end{gathered}
$$

The solution of this system of equations is

$$
\begin{gathered}
x=(1+\lambda)(1+\Delta) \frac{2 \lambda-\theta+\lambda \theta}{\theta(1-\lambda+2 \theta)^{2}}, \\
y=(1+\Delta) \frac{2 \lambda-\theta+\lambda \theta}{\theta(1-\lambda+2 \theta)} .
\end{gathered}
$$

Notice that

$$
y-x_{N}^{*}=\frac{2(\lambda-\theta)}{(1-\lambda+2 \theta) \theta}(1+\Delta)<0
$$

since $\lambda<\theta$ in the region of parameters under consideration, yielding the desired contradiction.

2.D. Suppose that investor 2 deviates by offering a mechanism $\mathcal{M}_{2}$ that entails full delegation.

Notice that, given the mechanism offered by investor 1, the advisor's ideal point over investor 2's asset allocation choice is

$$
\alpha_{2}^{*}\left(\sigma^{2}\right)=\left\{\begin{array}{lll}
\alpha_{N}^{A}\left(\sigma^{2}\right) & \text { if } \quad \sigma^{2} \leq x_{N}^{*} \\
\alpha_{E}^{A}\left(\sigma^{2}\right)-\theta \alpha_{N}^{*} & \text { if } \quad \sigma^{2}>x_{N}^{*}
\end{array} .\right.
$$

Hence: $\alpha_{N}^{A}\left(\sigma^{2}\right)<\alpha^{F}\left(\sigma^{2}\right)$ since $\theta>\lambda$ and

$$
\lambda \alpha^{F}\left(x_{N}^{*}\right)<\theta \alpha_{N}^{*} \quad \Rightarrow \quad \alpha_{E}^{A}\left(\sigma^{2}\right)-\theta \alpha_{N}^{*}<\alpha^{F}\left(\sigma^{2}\right) \quad \forall \sigma^{2}>x_{N}^{*} .
$$

But this implies that $\alpha_{2}^{*}\left(\sigma^{2}\right)<\alpha^{F}\left(\sigma^{2}\right)$ for all $\sigma^{2} \in \Sigma$. Then, by the same logic of the proof of Proposition 1, it follows that for investor 2 it is optimal to pool in a non-empty subset of $\Sigma$. A contradiction.

2.E. Finally, using the same arguments developed in the proof of cases $\mathbf{2 . A}, \mathbf{2 . B}$ and 2.C, it is easy to verify that deviations starting with a separating at $\sigma^{2}=1+\Delta$ and involving at least two disjoint pooling regions are not profitable.

The rest of the proof follows the same logic used in the proof of Proposition 2.

Proof of Corollary 1. We prove the result only for the exclusivity benchmark, the proof for the game with non-exclusivity follows exactly the same logic and is omitted for brevity. 
Suppose that the investor offers a very simple delegation mechanism to the advisor that requires him to choose the amount of wealth to allocate to the risky asset within the range $\alpha \in[\underline{\alpha}, \bar{\alpha}]$, with

$$
\underline{\alpha}=\pi \frac{1+\lambda}{1+\Delta}, \quad \bar{\alpha}=\pi \frac{1-\lambda}{1-\Delta} .
$$

For every $\sigma^{2}$, the advisor's optimization problem is

$$
\min _{\alpha \in[\underline{\alpha}, \bar{\alpha}]} \frac{1}{2}\left[\alpha-(1+\lambda) \alpha^{F}\left(\sigma^{2}\right)\right]^{2}
$$

The result then follows immediately since $\alpha_{E}^{A}\left(\sigma^{2}\right) \geq \bar{\alpha}$ if and only if $\sigma^{2} \leq x_{E}^{*}$, and $\alpha_{E}^{A}\left(\sigma^{2}\right) \geq \underline{\alpha}$ with equality only at $\sigma^{2}=1+\Delta$.

Proof of Proposition 4. To show this result we first need to compute the investors' expected utility with and without exclusivity.

Consider first the exclusivity benchmark. Using the result of Proposition 1 the investor's expected (indirect) utility is

$$
\begin{aligned}
\mathcal{W}_{E}^{*}=\frac{\gamma}{2 \Delta}\left[\int_{1-\Delta}^{x_{E}^{*}} \alpha_{E}^{*}\left[\pi-\frac{\alpha_{E}^{*} \sigma^{2}}{2}\right] d \sigma^{2}+\int_{x_{E}^{*}}^{1+\Delta} \alpha_{E}^{A}\left(\sigma^{2}\right)\left[\pi-\frac{\alpha_{E}^{A}\left(\sigma^{2}\right) \sigma^{2}}{2}\right] d \sigma^{2}\right]= \\
\frac{\gamma \pi^{2}}{2 \Delta}\left[\lambda+\frac{(1+\lambda)(1-\lambda)}{2} \ln \frac{(1+\Delta)(1-\lambda)}{(1-\Delta)(1+\lambda)}\right] .
\end{aligned}
$$

Next, consider the case of non-exclusive advice. Two cases must be distinguished depending on the type of equilibrium.

(1) In the region of parameters where there exist an equilibrium with partial delegation where both investors pool for low values of $\sigma^{2}$ and delegate otherwise - i.e., see Proposition 2 and the first part of Proposition 3 - the investors' expected utility is

$$
\begin{aligned}
& \mathcal{W}_{N}^{*}=\frac{\gamma}{2 \Delta}\left[\int_{1-\Delta}^{x_{N}^{*}} \alpha_{N}^{*}\left[\pi-\frac{\alpha_{N}^{*} \sigma^{2}}{2}\right]\right.\left.d \sigma^{2}+\int_{x_{N}^{*}}^{1+\Delta} \alpha_{N}^{A}\left(\sigma^{2}\right)\left[\pi-\frac{\alpha_{N}^{A}\left(\sigma^{2}\right) \sigma^{2}}{2}\right] d \sigma^{2}\right]= \\
& \frac{\gamma \pi^{2}}{2(1+\theta) \Delta}\left[\lambda-\theta+(1+\lambda) \frac{1-\lambda+2 \theta}{2(1+\theta)} \ln \frac{(1+\Delta)(1-\lambda+2 \theta)}{(1-\Delta)(1+\lambda)}\right]
\end{aligned}
$$

Notice that in this region of parameters $\mathcal{W}_{E}^{*}=\mathcal{W}_{N}^{*}$ for $\theta=0$ and that

$$
\frac{\partial \mathcal{W}_{N}^{*}}{\partial \theta}=\frac{\gamma \pi^{2}}{2 \Delta} \frac{(1+\lambda)}{(1+\theta)^{3}}\left[(\lambda-\theta) \ln \frac{(1+\Delta)(1-\lambda+2 \theta)}{(1-\Delta)(1+\lambda)}\right]>0
$$

since $\lambda>\theta$ and $\frac{(1+\Delta)(1-\lambda+2 \theta)}{(1-\Delta)(1+\lambda)}>1$. It then follows that $\mathcal{W}_{E}^{*} \geq \mathcal{W}_{N}^{*}$ if and only if $\theta \leq 0$.

(2) In the region of parameters where there exist an equilibrium with partial delegation where both investors pool for high values of $\sigma^{2}$ and delegate otherwise - i.e., see the second part of Proposition 3 
— the investors' expected utility is

$$
\begin{aligned}
\mathcal{W}_{N}^{*}=\frac{\gamma}{2 \Delta}\left[\int_{1-\Delta}^{x_{N}^{*}} \alpha_{N}^{A}\left(\sigma^{2}\right)\left[\pi-\frac{\alpha_{N}^{A}\left(\sigma^{2}\right) \sigma^{2}}{2}\right] d \sigma^{2}+\int_{x_{N}^{*}}^{1+\Delta} \alpha_{N}^{*}\left[\pi-\frac{\alpha_{N}^{*} \sigma^{2}}{2}\right] d \sigma^{2}\right]= \\
\frac{\gamma \pi^{2}}{2(1+\theta) \Delta}\left[\theta-\lambda+(1+\lambda) \frac{1-\lambda+2 \theta}{2(1+\theta)} \ln \frac{(1+\Delta)(1+\lambda)}{(1-\Delta)(1-\lambda+2 \theta)}\right] .
\end{aligned}
$$

Notice that $\mathcal{W}_{E}^{*}=\mathcal{W}_{N}^{*}$ for $\theta=\frac{2 \lambda}{1-\lambda}>\lambda$. Moreover,

$$
\frac{\partial \mathcal{W}_{N}^{*}}{\partial \theta}=\frac{\gamma(\lambda-\theta)(1+\lambda)}{(1+\theta)^{3}} \ln \frac{(1+\Delta)(1+\lambda)}{(1-\Delta)(1-\lambda+2 \theta)}<0
$$

since $\lambda<\theta$ and

$$
\frac{1+\lambda}{1-\lambda+2 \theta} \frac{1+\Delta}{1-\Delta}>1
$$

Hence, $\mathcal{W}_{E}^{*} \geq \mathcal{W}_{N}^{*}$ if and only if $\theta \geq \frac{2 \lambda}{1-\lambda}$.

Proof of Proposition 5. In the case of exclusivity, the average investment into the risky asset is

$$
\hat{\alpha}_{E}^{*}=\frac{1}{2 \Delta}\left[\int_{1-\Delta}^{x_{E}^{*}} \alpha_{E}^{*} d \sigma^{2}+\int_{x_{E}^{*}}^{1+\Delta} \alpha_{E}^{A}\left(\sigma^{2}\right) d \sigma^{2}\right]=\frac{\pi}{\Delta}\left[\lambda+\frac{(1+\lambda)}{2} \ln \frac{(1+\Delta)(1-\lambda)}{(1-\Delta)(1+\lambda)}\right] .
$$

By contrast, in the region of parameters in which there exists an equilibrium with partial delegation where both investors pool for low values of $\sigma^{2}$ and delegate otherwise - i.e., see Proposition 2 and the first part of Proposition 3 - the average investment into the risky asset is

$$
\hat{\alpha}_{N}^{*}=\frac{1}{2 \Delta}\left[\int_{1-\Delta}^{x_{N}^{*}} \alpha_{N}^{*} d \sigma^{2}+\int_{x_{N}^{*}}^{1+\Delta} \alpha_{N}^{A}\left(\sigma^{2}\right) d \sigma^{2}\right]=\frac{\pi}{\Delta(1+\theta)}\left[\lambda-\theta+\frac{1+\lambda}{2} \ln \frac{(1+\Delta)(1-\lambda+2 \theta)}{(1-\Delta)(1+\lambda)}\right] .
$$

Hence

$$
\hat{\alpha}_{E}^{*} \geq \hat{\alpha}_{N}^{*} \quad \Leftrightarrow \quad 2 \theta-\ln \left[\frac{1-\lambda+2 \theta}{1-\lambda}\left(\frac{(1-\Delta)(1+\lambda)}{(1+\Delta)(1-\lambda)}\right)^{\theta}\right] \geq 0 .
$$

The solution of $\hat{\alpha}_{N}^{*}=\hat{\alpha}_{E}^{*}$ with respect to $\Delta$ is

$$
\Delta_{1}=\frac{\frac{1+\lambda}{1-\lambda}\left(\frac{1-\lambda+2 \theta}{(1-\lambda) \exp 2 \theta}\right)^{\frac{1}{\theta}}-1}{\frac{1+\lambda}{1-\lambda}\left(\frac{1-\lambda+2 \theta}{(1-\lambda) \exp 2 \theta}\right)^{\frac{1}{\theta}}+1}
$$

where it can be checked that in the parameter region under consideration $\Delta_{1} \in(0,1)$. Notice also that

$$
\frac{\partial \ln \left[\frac{1-\lambda+2 \theta}{1-\lambda}\left(\frac{(1-\Delta)(1+\lambda)}{(1+\Delta)(1-\lambda)}\right)^{\theta}\right]}{\partial \Delta} \geq 0 \quad \Leftrightarrow \quad \theta \leq 0
$$

which directly implies the result.

Consider now the region of parameters where there exists an equilibrium with partial delegation 
where both investors pool for high values of $\sigma^{2}$ and delegate otherwise - i.e., see the second part of Proposition 3 - the average investment into the risky asset is

$$
\hat{\alpha}_{N}^{*} \equiv \frac{1}{2 \Delta}\left[\int_{1-\Delta}^{x_{N}^{*}} \alpha_{N}^{A}\left(\sigma^{2}\right) d \sigma^{2}+\int_{x_{N}^{*}}^{1+\Delta} \alpha_{N}^{*} d \sigma^{2}\right]=\frac{\pi}{\Delta(1+\theta)}\left[\theta-\lambda+\frac{1+\lambda}{2} \ln \frac{(1+\Delta)(1+\lambda)}{(1-\Delta)(1-\lambda+2 \theta)}\right] .
$$

Hence

$$
\hat{\alpha}_{E}^{*} \geq \hat{\alpha}_{N}^{*} \quad \Leftrightarrow \quad 2(2 \lambda-\theta(1-\lambda))-(1+\lambda) \ln \left[\frac{(1+\lambda)^{(2+\theta)}}{(1-\lambda+2 \theta)(1-\lambda)^{\theta}}\left(\frac{1-\Delta}{1+\Delta}\right)^{\theta}\right]>0 .
$$

The solution of $\hat{\alpha}_{N}^{*}=\hat{\alpha}_{E}^{*}$ with respect to $\Delta$ is

$$
\Delta_{2}=\frac{\left[\frac{(1-\lambda+2 \theta)(1-\lambda)^{\theta}}{(1+\lambda)^{(2+\theta)}} \exp \left(\frac{2(2 \lambda-\theta+\lambda \theta)}{1+\lambda}\right)\right]^{-\frac{1}{\theta}}-1}{\left[\frac{(1-\lambda+2 \theta)(1-\lambda)^{\theta}}{(1+\lambda)^{(2+\theta)}} \exp \left(\frac{2(2 \lambda-\theta+\lambda \theta)}{1+\lambda}\right)\right]^{-\frac{1}{\theta}}+1}<1
$$

Suppose that $\Delta_{2}>0$. Then,

$$
\frac{\partial \ln \left[\frac{(1+\lambda)^{(2+\theta)}}{(1-\lambda+2 \theta)(1-\lambda)^{\theta}}\left(\frac{1-\Delta}{1+\Delta}\right)^{\theta}\right]}{\partial \Delta}<0
$$

since $\theta>0$ in the parameter region under consideration. This implies that $\alpha_{E}^{*} \geq \alpha_{N}^{*}$ if and only if $\Delta \geq \Delta_{2}$. We now show under which conditions $\Delta_{2}>0$. Notice that this requires

$$
\frac{(1-\lambda+2 \theta)(1-\lambda)^{\theta}}{(1+\lambda)^{(2+\theta)}} \exp \left(\frac{2(2 \lambda-\theta+\lambda \theta)}{1+\lambda}\right)<1
$$

which implies

$$
\Phi(\lambda, \theta) \equiv \ln \frac{(1-\lambda+2 \theta)(1-\lambda)^{\theta}}{(1+\lambda)^{(2+\theta)}}+\frac{2(2 \lambda-\theta+\lambda \theta)}{1+\lambda}<0 .
$$

Notice that $\Phi(\lambda=0, \theta)=-2 \theta+\ln (2 \theta+1)<0$ for each $\theta \in[0,1]$ and $\Phi(\lambda=\theta, \theta)=\ln \frac{(1-\theta)^{\theta}}{(1+\theta)^{(1+\theta)}}+2 \theta<$ 0 if and only if $\theta>0.537$. It is then easy to show that there exists a threshold $\lambda^{*}<\theta$ such that: $(i)$ if $\lambda>\lambda^{*}$ then $\Phi(\lambda, \theta)<0$ for every $\theta$; (ii) if $\lambda \leq \lambda^{*}$ there exists a function $\theta(\lambda)$, which solves $\Phi(\lambda, \theta)=0$, such that $\Phi(\lambda, \theta)<0$ if and only if $\theta>\theta(\lambda)$. Hence, $\Delta_{2}>0$ if: (i) $\lambda \geq \lambda^{*}$; (ii) $\lambda<\lambda^{*}$ and $\theta>\theta(\lambda)$.

\section{References}

[1] Admati, A.R and P. Pfleiderer, (1997), "Does it All Add Up? Benchmarks and the Compensation of Active Portfolio Managers," Journal of Business, 70: 323-50.

[2] Allen, F., (1985), "Contracts to Sell Information," Rodney L. White Center for Financial Research Working Papers 6-87, Wharton School Rodney L. White Center for Financial Research.

[3] Allen, F., And G. Gordon, (1993), "Churning Bubbles," Review of Economic Studies, 60: 813-836. 
[4] Alonso, R., And N. Matouschek, (2008), "Optimal Delegation," Review of Economic Studies, 75: 259-293.

[5] Asparouhova, E., P. Bossaerts, J. Copic, B. Cornell, J. Cvitanic, and D. Meloso, (2013), "Experiments on Asset Pricing under Delegated Portfolio Management," forthcoming Managment Science.

[6] Attar, A., T. Mariotti and F. Salanié, (2011), "Nonexclusive Competition in the Market for Lemons," Econometrica, 79: 1869-1918.

[7] Bergstresser, Daniel, John Chalmers, and Peter Tufano, (2009), "Assessing the Costs and Benefits of Brokers in the Mutual fund Industry", Review of Financial Studies, 22: 41294156.

[8] Bhattacharya, S., And P. Pfleiderer, (1985), "Delegated Portfolio Management," Journal of Economic Theory, 36: 1-25.

[9] Calcagno, R., and C. Monticoni, (2013), "Financial Literacy and the Demand for Financial Advice," mimeo.

[10] Chalmers, J., And J. Reuter, (2012), "What is the Impact of Financial Advisors on Retirement Portfolio Choices and Outcomes?", mimeo.

[11] Chater, N., S. Huck, and R. Inderst, (2010), "Consumer Decision-making in Retail Investment Services: a Behavioral Economics Perspective", Report to the European Commission/SANCO.

[12] Chen, H., and G. G. Pennacchi, (2009), "Does Prior Performance Affect a Mutual Fund's Choice of Risk? Theory and Further Empirical Evidence", Journal of Financial and Quantitative Analysis, 44: 745-775.

[13] Chevalier, J., And G. Ellison, (1997), "Risk Taking by Mutual Funds as a Response to Incentives," Journal of Political Economy, 105: 1167-1200.

[14] Crawford, V. P., And J. Sobel, (1982), "Strategic Information Transmission," Econometrica, 50: $1431-1451$

[15] Das, S. R., and R. K. Sundaram, (2002), "Fee Speech: Signaling, Risk-Sharing, and the Impact of Fee Structures on Investor Welfare," Review of Financial Studies 15, 1465-1497.

[16] Del Guercio, D., J. Reuter, P. Tkac, (2010), "Broker Incentives and Mutual Fund Market Segmentation", mimeo.

[17] Dessein, W., (2002), "Authority and Communication in Organizations", Review of Economic Studies, 69: 811-838.

[18] Farrel J., And R. Gibbons, (1989), "Cheap Talk with Two Audiences", American Economic Review, 79: 1214-1223.

[19] Foerster, S., Linnainmaa, J. T., Melzer, B. T. and A. Previtero, (2014), "Retail Financial Advice: Does One Size Fit All?", mimeo.

[20] Gennaioli, N., A. Shleifer and R. Vishny, (2013), "Money Doctors," Journal of Finance, forthcoming.

[21] Georgarakos, D., and R. Inderst, (2011), "Financial Advice and Stock Market Participation", mimeo.

[22] Gruber, M., (1996), "Another Puzzle: the Growth in Actively Managed Mutual Funds," Journal of Finance, 51: 783-810.

[23] Guiso, L., And Viviano, E., (2013), "How Much Can Financial Literacy Help?", EIEF Working Papers Series 1325. 
[24] Hackethal, A., M. Haliassos, and T. Jappelli, (2012), "Financial Advisors: A Case of Babysitters?," Journal of Banking and Finance, 36: 509-524,

[25] Hung, A., C. Noreen, J. Dominitz, E. Talley, C. Berrebi and F. Suvankulov, (2008), "Investor and Industry Perspectives on Investment Advisers and Broker-Dealers," Technical Report, Rand Institute for Civil Justice.

[26] Holmström, B., (1984), "On the Theory of Delegation", in M. Boyer and R. Kihlstrom (eds.), Bayesian Models in Economic Theory, Elsevier.

[27] Inderst, R., and M. Ottaviani, (2012a), "How (not) to Pay for Advice: A Framework for Consumer Financial Protection," Journal of Financial Economics, 105: 393-411.

[28] Inderst, R., And M. Ottaviani, (2012b), "Financial Advice," Journal of Economic Literature, 50: 494-512.

[29] Kaniel, R., and P. Kondor, (2013), "The Delegated Lucas Tree," Review of Financial Studies, 26: 929-984.

[30] Kolotilin, A., H. Li and W.Li, (2013), "Optimal Limited Authority for Principal," Journal of Economic Theory, 148: 2344-2382.

[31] Malkiel, B.G., (1995), "Returns From Investing in Equity Mutual Funds 1971-1991," Journal of Finance, 50: 549-570.

[32] Malmendier, U., And Nagel, S., (2011), "Depression Babies: Do Macroeconomic Experiences Affect Risk Taking?", Quarterly Journal of Economics, 126: 373-416.

[33] Martimort, D., And A. Semenov, (2006), "Continuity in Mechanism Design without Transfers," Economic Letters, 93: 182-189.

[34] Martimort, D., And L. Stole, (2002), "The Revelation and Delegation Principles in Common Agency Games," Econometrica, 70: 1659-1673.

[35] Martimort, D., And L. Stole, (2003), "Contractual Externalities and Common Agency Equilibria," The B.E. Journal of Theoretical Economics, 3: 1-40.

[36] Melumad, N., and T. Shibano, (1991), "Communication in Settings with no Transfers," RAND Journal of Economics, 22: 437-455.

[37] Monti, M., V. Pelligra, L. Martignon and N. Berg, (2014), "Retail investors and financial advisors: New evidence on trust and advice taking heuristics", Journal of Business Research, 67: $1749-1757$.

[38] Morgan, J., And P. Stocken, (2003), "An Analysis of Stock Recommendations," RAND Journal of Economics, 34: 183-203.

[39] Ottaviani M., (2000), "The Economics of Advice," mimeo.

[40] Palomino, F. and A. Prat, (2003), "Risk Taking and Optimal Contracts for Money Managers," RAND Journal of Economics, 34: 113-37.

[41] Palomino, F. and H. Uhlig, (2007), "Should Smart Investors Buy Funds with High Returns in the Past?," Review of Finance, 11: 51-70.

[42] Pavan, A., And G., Calzolari, (2009), "Sequential Contracting with Multiple Principals," Journal of Economic Theory, 144: 503-531.

[43] Stoughton, N., (1993), "Moral Hazard and the Portfolio Management Problem," Journal of Finance, 48: 2009-2028.

[44] Stracca, L. (2005), "Delegated Portoflio Management: a Survey of the Theoretical Literature," ECB Working paper series, N. 520/Sep. 2005. 
[45] Tversky, A. and Kahneman, D., (1974), "Judgment under uncertainty: Heuristics and biases", Science, 185: 1124-1131. 